\title{
OPINIONE PUBBLICA E POLITICA ESTERA IN ITALIA: IL CASO DELLA BOSNIA
}

\author{
di Paolo Bellucci e Pierangelo Isernia
}

\section{Introduzione}

La guerra contro la Repubblica Federale di Jugoslavia ha evidenziato i problemi della politica estera italiana degli anni '90. Con non più del $40 \%$ dell'opinione pubblica stabilmente a favore dei raid aerei contro la Serbia ed il Kosovo, una veemente opposizione del Vaticano e del Papa in prima persona ed una maggioranza di governo divisa al suo interno tra negoziatori ad oltranza ed auspici di una immediata escalation terrestre, si riproponeva, con maggiore evidenza del passato, il ridotto margine di autonomia dell'esecutivo nel settore della politica di sicurezza. A poco meno di due anni dalla crisi albanese, la leadership politica italiana si è trovata così ad affrontare l'ennesima prova di politica estera, per giunta sul terreno delle armi, un terreno sul quale nell'ultimo quarantennio repubblicano raramente un governo si era avventurato. Rispetto all'ambiente tut-

Versioni precedenti di questo saggio sono state presentate al Workshop «Public Opinion and the Bosnia crisis» della International Society for Political Psychology, 18-20 luglio 1997, Cracovia, Polonia, e alla 27th Joint Session of Workshops, Workshop on «Democracy. Public Opinion and the Use of Force in a Changing International Environment» dell'European Consortium for Political Research, 23-27 aprile 1998, Warwick, Regno Unito. Desideriamo ringraziare la Swg-Servizi Integrati di Ricerca. Trieste per averci fornito $i$ risultati delle loro inchieste sulla Bosnia, Polimetrica Srl per l'utilizzo del Bosnia Tracking Poll e dell'inchiesta Difebarometro n. 4, Rudy Perina e Howald Ciaccone del Dipartimento di Stato di Washington, il ministro Shaun Burns dell'Ambasciata degli Stati Uniti a Roma, la dott.ssa Antonella Deledda e lo staff dell'Ufficio ricerche e documentazione, Dipartimento internazionale della Camera dei deputati, $i$ responsabili dell'emittente televisiva Telepace ed in particolare il dott. Aldo Schiavazzi. Ringraziamo infine l'United States Information Agency - in particolare il direttore dell'Office of Research, Ann Pincus, e l'allora direttore dell'European Branch, Mary MacIntosh, nonché Susan White - per avere reso disponibili $i$ dati dell'Usia relativi all'Italia. Ringraziamo inoltre Reinbard Moschner $e$ Peter Schubert del Zentral Archive di Colonia, che ci hanno fornito $i$ dati Euroflash sulla Bosnia.

RIVISTA ITALIANA DI SCIENZA POLITICA / a. XXIX, n. 3, dicembre 1999 
to sommato «placido» nel quale la politica estera italiana ha operato nel secondo dopoguerra, gli anni ' 90 , con un ininterrotto susseguirsi di crisi (dal Golfo alla Somalia, dall'Albania alla Bosnia, per finire con il Kosovo) hanno prodotto un maggior numero di sfide, in aree molto più vicine e rilevanti per gli interessi nazionali italiani e in un quadro di minore possibilità di far ricorso al proprio tradizionale alleato, gli Stati Uniti, per risolvere i propri fondamentali problemi di sicurezza. Da qui la necessità di costruire un consenso nazionale intorno alle scelte del governo e, di conseguenza, il crescente interesse per il ruolo che l'opinione pubblica assume nella politica estera italiana. Il Kosovo tuttavia non è il primo caso in cui l'opinione pubblica entra nei calcoli dei decisori nazionali. In questo saggio intendiamo esplorare questo ruolo in un'altra recente crisi che ha visto coinvolta l'Italia, quella relativa al dissolvimento della ex-Jugoslavia, con particolare riferimento alla crisi in Bosnia-Herzegovina. Come diremo nelle conclusioni, da questa esperienza è possibile trarre alcune considerazioni che sembrano dimostrarsi valide anche nel caso del Kosovo.

La valutazione del ruolo dell'opinione pubblica in politica estera è cambiata radicalmente rispetto ad alcuni anni fa. Sino ai primi anni '80, era diffusa la convinzione che l'opinione pubblica avesse, in questioni di politica internazionale, una scarsa influenza e che questo fosse un bene, data la mutevolezza e instabilità degli atteggiamenti del pubblico su temi per i quali ha scarso interesse e ben poche conoscenze. Nel corso di un decennio si è assistito ad un radicale rovesciamento di orientamenti e molte delle tradizionali convinzioni sulla natura, le caratteristiche e l'impatto del pubblico sulle politiche pubbliche sono state scosse. La tesi, avanzata da Almond $\left(1960^{2}\right)$ e confermata da Rosenau (1961), di una sostanziale volatilità del pubblico su questi temi, è stata prima criticata metodologicamente (Caspary 1970) e poi, utilizzando una più sistematica raccolta di dati, sostantivamente (Page e Shapiro 1983; 1992; Shapiro e Page 1988), sino a concludere che essa era sostanzialmente un «mito». Anche lo scetticismo sulle capacità cognitive e sulla scarsa presa delle ideologie sul pubblico americano sintetizzato da Converse (1964) è stato quindi scosso da più approfondite analisi, dirette a mostrare $i$ limiti metodologici (Achen 1975) e storici (Nie e Kristi 1974) delle tesi di Converse, portando a rivalutare, in un più sobrio quadro di riferimento teorico, le capacità cognitive del pubblico americano (Hurwitz e Peffley 
1987; Kinder e Sears 1985). Infine, il drastico giudizio sull'incapacità del pubblico di farsi sentire in questioni concernenti la pace e la guerra è stato sottoposto a revisione, sulla base sia di analisi longitudinali (Page e Shapiro 1983; Page, Shapiro e Dempsey 1987) che di approfonditi studi di caso (Jacobs 1993; Jacobs e Shapiro 1994), sia di ricerche volte ad accertare direttamente il peso dell'opinione pubblica nei calcoli decisionali di politici e funzionari (Kull, Destler e Ramsay 1997; Herbst 1998; Geer 1996).

Sfortunatamente, gran parte di questo attivismo e dei risultati che ne sono scaturiti concerne gli Stati Uniti e la loro esperienza. Vi è una preoccupante mancanza di analisi comparate (Holsti 1992, 460) e questo, per la natura del sistema politico americano e per la sua posizione di grande superpotenza, inficia la generalizzabilità dei risultati scientifici sinora ottenuti. Inoltre, come diversi studiosi hanno mostrato, l'opinione pubblica può avere un ruolo differente a seconda dei contesti politici ed istituzionali (Risse-Kappen 1991; Cohen 1995; Panebianco 1997). Solo da pochi anni si è cominciato a dedicare una maggiore attenzione alla natura delle relazioni tra opinione pubblica e politica estera nel caso dei paesi europei, anche se in prevalenza nel settore del processo di integrazione europea (si veda per esempio Niedermayer e Sinnott 1995; Gabel 1998; Eichenberg 1998, nonché Everts e Isernia in stampa).

Nella vicenda della Bosnia, l'opinione pubblica è stata spesso menzionata, a volte tenuta in conto $\mathrm{e}$, forse più raramente, temuta dai politici e burocrati italiani chiamati di volta in volta a decidere quale posizione il nostro paese doveva tenere di fronte agli sviluppi della crisi balcanica. Il caso che presentiamo come apertura di questo saggio illustra, simbolicamente ed insieme concretamente, una occasione di interazione tra opinione pubblica e decisori politici in cui emergono alcune caratteristiche della natura di questo rapporto.

\section{Il ministro e l'opinione pubblica}

Alla fine del 1993 la guerra in Bosnia, con il suo succedersi di cessate-il-fuoco falliti, di piani di pace, di bombardamenti serbi su Sarajevo e di violenze indiscriminate sulla popolazione civile, continuava senza che vi fosse alcun segnale che potesse essere controllata o fatta cessare. Anzi, tra il novembre e il di- 
cembre 1993 vi erano crescenti segni di insofferenza da parte di inglesi e francesi per il rapido deterioramento della situazione in Bosnia-Herzegovina, che si tradussero in manifeste minacce dei governi dei due paesi di ritirare le loro truppe. In questa situazione, il 23 dicembre 1993 il ministro degli Esteri italiano, Beniamino Andreatta, concesse un'intervista alla Tv privata cattolica Telepace. L'intervista fu mandata in onda il $1^{\circ}$ gennaio 1994 e una nota con i suoi contenuti fu inviata a tutti i principali giornali. In essa, Andreatta prendeva nettamente posizione contro la tesi vaticana, riproposta nuovamente alcuni giorni prima dal Segretario di stato, secondo la quale un intervento militare per ragioni umanitarie, allo scopo di prevenire il genocidio e la «pulizia etnica» di intere popolazioni in paesi stranieri era moralmente legittimo. Andreatta non criticava l'argomento in quanto tale, riconoscendo la legittimità morale di una tale forma di intervento, ma sosteneva che questo tipo di azione era impensabile nelle moderne democrazie. Queste ultime sono dominate dall'opinione pubblica ed essa era nettamente contraria ad ogni forma di intervento armato. Il ministro sosteneva che una delle ragioni per cui i governi Europeo-occidentali erano riluttanti a farsi coinvolgere nella crisi balcanica era proprio l'opposizione dell'opinione pubblica all'intervento militare. Per usare le parole dello stesso Andreatta - come riportate tra virgolette un mese più tardi, citando alcune frasi della nota per la stampa di Telepace, dal quotidiano «la Repubblica» in un articolo non firmato dal titolo Ma chi vuole l'intervento - «Le nostre popolazioni non ci danno, né negli Stati Uniti né in Europa, un mandato ad usare le armi per riportare la giustizia. Questo è il dramma di coloro che sono coinvolti nella crisi in Bosnia» e aggiungeva «molte delle responsabilità per il fallimento dei paesi occidentali nella questione balcanica dipendono dall'opinione pubblica».

Nella sua intervista televisiva, così come ci è stato confermato dal giornalista che la condusse, Andreatta non aveva tuttavia menzionato alcuna cifra a sostegno della sua tesi. Al contrario, «la Repubblica» del 26 gennaio 1994 che riprese quell'intervento, dopo aver citato tra virgolette le frasi di Andreatta sopra riportate, si riferiva, a conferma delle ipotesi di Andreatta, a «un sondaggio realizzato su un campione di 800 italiani dalla Swg (e pubblicato da «Famiglia Cristiana»). I dati rivelano che il $61 \%$ degli intervistati non vogliono «che nella ex-Jugoslavia guerra si aggiunga alla guerra» e, perciò, si oppongono a qualsiasi forma 
di intervento armato» («la Repubblica», 26 gennaio 1994, p. 10). Il quotidiano non riportava nell'articolo né la data in cui era stata condotta l'inchiesta, né, egualmente importante, la formulazione della domanda. In realtà, come vedremo tra un istante, entrambi questi elementi erano cruciali perché fornivano un'immagine leggermente diversa da quella proiettata dal giornale. Per finire con Andreatta, alcuni giorni dopo, questa volta a Vienna, il ministro reiterò la sua tesi che molti dei fallimenti europei in Bosnia erano da attribuire all'opinione pubblica dell'Europa occidentale e degli Stati Uniti. Può essere interessante ricordare che agli inizi di febbraio, probabilmente sotto la pressione degli eventi (l'uccisione di tre giornalisti a Mostar, una forte dichiarazione papale a favore dell'intervento e il bombardamento del mercato di Sarajevo che uccise 68 persone e ne ferì 197), lo stesso ministro non solo si dichiarò esplicitamente favorevole all'intervento militare della Nato («la Repubblica», 6 febbraio 1994) ma ridimensionò il ruolo dell'opinione pubblica nelle scelte di politica estera del nostro paese («la Repubblica», 13 febbraio 1994).

Questa storia è, a nostro avviso, ricca di suggestioni e informazioni sulla natura dei rapporti tra decisori e opinione pubblica, sull'influenza dei mass media in questo rapporto, e sul ruolo che le inchieste di opinione giocano in queste interazioni. Cominciamo dai media, ed in particolare dal modo in cui il quotidiano utilizzò le fonti dei sondaggi. Quello da noi riportato è infatti un caso esemplare di cattivo uso dei sondaggi': le informazioni provenienti da quel sondaggio non giustificavano infatti le affermazioni del giornalista. Due in particolare sono i fattori rilevanti. Il primo è la data dell'inchiesta, condotta dalla Swg il 4 agosto 1993 e riportata da «Famiglia Cristiana» più di 5 mesi prima dell'articolo del quotidiano romano, il che pone seri dubbi sul fatto che i dati riflettessero l'atteggiamento del pubblico all'inizio del 1994. Il secondo fattore è ancora più cruciale: non vi è dubbio che il $61 \%$ degli intervistati era contraria, il $28 \%$ favorevole e l' $11 \%$ non voleva esprimere un opinione, ma a che cosa? Qual era la domanda che generava questa distribuzione? La domanda era questa: «A questo punto della vicenda in Bosnia, lei sarebbe favorevole o contrario ad un intervento

1 Nella letteratura politologica e sociologica italiana vi è ancora scarso interesse per il rapporto tra stampa e sondaggi. Per un'eccezione Leiss e Paolozzi (1997). 
armato, sapendo che le forze di pace andrebbero incontro a perdite umane e a grosse spese militari?». Si trattava quindi non solo di una domanda fortemente «viziata» in direzione di una risposta negativa, ma che, soprattutto, non faceva nessun riferimento all'idea di una partecipazione italiana all'intervento armato. In realtà, i dati di inchieste già disponibili all'epoca rivelavano con chiarezza che l'orientamento generale dell'opinione pubblica sulla questione bosniaca era esattamente opposto a quello percepito dal ministro degli Esteri italiano e riportato dal giornale: una stabile maggioranza assoluta degli italiani era a favore non solo di un più deciso intervento armato, ma anche della partecipazione italiana ad un simile tipo di missione, ed una consistente maggioranza relativa era a favore di tale intervento anche in caso di perdite umane tra le truppe.

Passando ai politici, egualmente interessante è, ai nostri fini, stabilire che cosa avesse in mente il nostro ministro degli Esteri quando diceva che l'opinione pubblica italiana si opponeva a un intervento militare in Bosnia. Pensava alle opinioni del pubblico in generale, alla «massa» insomma, o all'opinione pubblica come è rappresentata dai e nei mass media, ai suoi colleghi in parlamento, a quelli del suo partito o agli esperti militari e civili? Si trattava di un caso di misreading ${ }^{2}$ degli orientamenti del pubblico o di un esplicito - e ben riuscito - tentativo di manipolarli, con l'aiuto volontario o involontario della stampa, allo scopo di rafforzare la sua posizione? Non disponiamo delle informazioni necessarie per rispondere conclusivamente a queste domande, tuttavia lo svolgersi degli eventi ci consente alcune considerazioni, da intendere comunque come disciplined guesses piuttosto che come conclusioni.

Alcuni elementi vanno tenuti presenti in questa storia. Il primo è che le forze armate italiane e, in generale, gli esperti di questioni militari erano tendenzialmente contrari alla partecipazione italiana in un'operazione militare in Bosnia, per ragioni sia tecniche che politiche. L'Italia si era già trovata in difficoltà in Somalia e la situazione in Bosnia era considerata ancora più difficile, in un contesto in cui le regole di ingaggio dettate dalle Nazioni unite contribuivano ad indebolire la posizione dei militari schierati. In secondo luogo, i mass media in generale, e gli

2 Per una affascinante ed innovativa analisi della tendenza dei politici a percepire erroneamente la distribuzione delle opinioni del pubblico e delle sue cause si veda Kull, Destler e Ramsay (1998). 
editorialisti in particolare, erano pronti a denunciare la passività dei governi occidentali di fronte al massacro in Jugoslavia, ma molto più riluttanti a suggerire cosa fare e ancor meno favorevolmente orientati ad un intervento delle truppe italiane. Il consenso generale nella stampa era che gli Stati Uniti fossero il solo attore in grado di intervenire efficacemente per fermare i combattimenti, una convinzione, per inciso, in linea con quella della maggioranza del pubblico italiano ${ }^{3}$. Terzo, Andreatta era presumibilmente consapevole dell'opposizione all'intervento all'interno del suo partito, il Ppi. In una inchiesta sui membri delle commissioni esteri e difesa di Camera e Senato della primavera 1995, Bellucci (1998) aveva posto due domande sulla crisi bosniaca identiche a quelle sottoposte a campioni rappresentativi della popolazione italiana nel dicembre 1994 e nel luglio 1995. Da questi dati emergeva che, nel complesso, l'opinione pubblica era leggermente più favorevole dell'élite parlamentare sia ad una missione Nato che alla partecipazione italiana a essa. Cruciali erano tuttavia le differenze tra le preferenze degli elettori e quelle dei parlamentari dei corrispondenti partiti: la distanza tra elettori ed élites parlamentari era massima proprio per Rifondazione Comunista e Ppi. Tra i parlamentari del Ppi, il 60\% era contrario all'invio di truppe italiane in Bosnia, mentre tra gli elettori di questo partito il $60 \%$ era favorevole. La minore popolarità di un intervento armato tra i parlamentari cattolici potrebbe contribuire a spiegare, almeno in parte, le dichiarazioni di Andreatta. In sostanza, nel menzionare in varie occasioni la riluttanza del pubblico ad un intervento armato, il ministro poteva ben avere in mente non tanto l'opinione pubblica come viene misurata dalle inchieste di opinione, quanto quella che emerge dai mass media e quella espressa dai parlamentari del suo partito di riferimento.

Alla luce di queste considerazioni, il riferimento del ministro degli affari esteri italiano all'opinione pubblica può essere una conseguenza delle contrastanti pressioni cui era sottoposto. Andreatta era preso in mezzo a due fuochi, tra coloro che, nel suo partito in primo luogo, ma anche nell'amministrazione centrale, si opponevano strenuamente ad un intervento armato del-

3 Nel maggio-giugno 1994 il 55\% degli italiani riteneva che gli Stati Uniti avessero un ruolo importante da giocare per far cessare la guerra. Se si escludono Nazioni unite e Nato, nessuno stato od organizzazione era ritenuto più adatto a far cessare la guerra. 
l'Italia da un lato e coloro che, in Vaticano e tra i partiti di sinistra che pur sostenevano il governo, ritenevano che il governo italiano stesse facendo ben poco per aiutare a risolvere la situazione in Bosnia. Se a questo si aggiunge che, probabilmente, le sue preferenze personali andavano a favore dell'intervento, gettare la colpa della riluttanza governativa sull'opinione pubblica era una maniera elegante sia per non dover esporre preferenze personali in contrasto con quelle del suo partito, sia per attribuire la posizione ufficiale del governo (contro la partecipazione italiana) al clima generale di opinione. In poche parole, Andreatta scelse quella che nel gergo della teoria dei giochi si chiama una strategia delle «mani legate» (Schelling 19807; Putnam 1988). Infine, in un gioco di specchi - tipico del circuito opinione pubblica, mass media, decisori (Mc Combs et al. 1991) il fatto che le sue dichiarazioni fossero riprese, ampliate e sostanziate dai mass media rafforzava ulteriormente la sua posizione, trasformando in realtà effettuale quelle che, inizialmente, erano solo enunciazioni verbali e rendendo agli spettatori plausibile la stessa realtà che quelle dichiarazioni avevano contribuito a creare in prima istanza. In questa specifica occasione, ed in generale per l'intera durata della crisi bosniaca, i governanti italiani non fecero alcuno sforzo per capire quale fosse effettivamente l'umore del paese. Operare sotto questo «velo di ignoranza», comunque, aiutava $\mathrm{i}$ governanti a perseguire una linea di policy in linea con le loro preferenze (o forse più propriamente con l'equilibrio delle forze all'interno del governo e dell'amministrazione) - quella di non fare niente - accusando allo stesso tempo l'opinione pubblica per la loro impotenza. Insomma, l'opinione pubblica era un modo elegante per nascondere la loro mancanza di volontà di impegnare le truppe in Bosnia.

\section{Opinione pubblica e politica estera in Italia}

In Italia è abbastanza diffusa tra commentatori, politici e studiosi la convinzione che l'opinione pubblica non abbia alcun ruolo nella politica estera italiana. Nella suggestiva (ma forse sbrigativa) frase di Sereno (cit. in Kogan 1965, 40): «gli italiani amano il cinema, il calcio e le ragazze. Non sono interessati alla politica e ancor meno alla politica estera». Questa immagine degli italiani, vagamente «orientalista», è stata per molti anni rafforzata da una serie di studi sia qualitativi che quantitativi con- 
dotti alla metà degli anni ' 50 ed è restata con noi sino ad oggi (Putnam 1993) ${ }^{4}$. Secondo questa interpretazione, la mancanza di interesse per e di conoscenza della realtà internazionale produce un'opinione pubblica così volatile e umorale da renderla totalmente inaffidabile come seria risorsa politica. Dall'altro canto, l'esistenza di partiti forti, che monopolizzano i canali di comunicazione tra pubblico, governo ed amministrazione e che si costituiscono come gli interpreti principali degli umori dell'opinione pubblica, rende tutto sommato marginale il ruolo di questa nelle politiche pubbliche italiane. $\mathrm{Ma}$ in che misura questa immagine del sistema politico italiano è ancora valida al giorno d'oggi? Per rispondere a questa domanda occorre brevemente $^{5}$ discutere la natura dell'opinione pubblica italiana ed il suo impatto sul policy making.

La natura dell'opinione pubblica italiana. In che misura l'immagine di disinteresse, ignoranza e volatilità dell'opinione pubblica italiana è corroborata dai dati empirici disponibili? Se si ha la pazienza di esaminare il sorprendentemente ${ }^{6}$ ricco materiale empirico disponibile si trova che, contrariamente all'«eccezionalismo» coltivato dai nostri osservatori, l'opinione pubblica italiana non è dissimile da quella degli altri paesi occidentali. Più precisamente: l'opinione pubblica italiana non è al giorno d'oggi radicalmente differente da quella di altri paesi nel livello di attenzione, interesse e conoscenza dei problemi internazionali, e non è particolarmente erratica o volatile nei suoi orientamenti.

Ovviamente anche in Italia sono i problemi interni, e in particolare quelli economici (disoccupazione) e politici (violenza e instabilità governativa), a suscitare le maggiori preoccupazioni.

4 Può essere interessante ricordare che mentre la scienza politica americana è sempre stata incline a spiegare il caso italiano (e le sue «anomalie») con riferimento a variabili culturali, e specificamente, con l'assenza di spirito civico, gli studiosi italiani hanno sempre preferito guardare a quelle strutturali. Una visione più articolata del complesso intreccio di relazioni tra istituzioni politiche e cultura civica è in Putnam (1993), che comunque arriva a conclusioni sostanzialmente analoghe a quelle di studi precedenti. Per una interpretazione «orientalista» di questa corrente di studi di provenienza prevalentemente anglosassone, ma non solo (Tullio-Altan 1986), si veda Agnews (1997).

5 Per una analisi più approfondita ci permettiamo di rimandare a Bellucci e Isernia (in stampa).

6 In rapporto al livello altrettanto sorprendentemente modesto di attenzione dedicato a questi temi dai mass media e dagli studiosi accademici. Per un'eccezione si veda Guidorossi (1984). 
$\mathrm{Ma}$ in questo gli italiani non sono differenti dagli altri popoli. La mancanza di interesse in politica estera, menzionata come caratteristica peculiare del nostro paese (Kogan 1965; Graziano 1968), era forse vera negli anni ' 50 , ma lo è sicuramente meno già dagli anni '60 e '70, per effetto del processo di modernizzazione socio-culturale che ha accompagnato lo sviluppo economico del nostro paese (Sciolla 1990). Inoltre la realtà politica, per definizione una realtà «mediata», un unobtrusive issue (McCombs, Einsiedel e Weaver 1991), non si presta ad essere direttamente percepita dal singolo, ma piuttosto ad essere assorbita tramite i mezzi di comunicazione di massa. Da questo punto di vista, tra gli anni '50 e gli anni '80 la crescente scolarizzazione di massa ed il costituirsi di un sistema dei media nazionale hanno contribuito a omogeneizzare il pubblico italiano a quello degli altri paesi occidentali'. Per effetto di questi processi, l'opinione pubblica italiana degli anni '80 mostra verso questioni come le armi nucleari, gli aiuti allo sviluppo, i movimenti per la pace livelli di attenzione analoghi a quelli di altri paesi occidentali (Battistelli e Isernia 1992; Isernia 1996b). Ovviamente, gli italiani hanno scarse conoscenze della realtà internazionale, come peraltro i cittadini degli altri paesi europei e gli stessi americani (Delli Carpini e Keeter 1992). Tra il 1955 ed il 1980 non più del $70 \%$ degli italiani intervistati aveva sentito parlare della Nato, ma, se può consolarci, negli anni '50 Almond (1960) stimava che non più del $10 \%$ degli americani aveva qualche conoscenza delle questioni internazionali e Converse (1964) ha calcolato che l'ammontare di «ideologi» (in senso molto lato) tra gli americani non supera il $5 \%$.

Non solo l'opinione pubblica italiana non è così abissalmente differente, in termini di conoscenza, interesse ed attenzione per le questioni internazionali, da quella degli altri paesi occidentali, ma non è peraltro nemmeno più volatile. Sulla base dell'esame di più di 8.000 domande poste in inchieste condotte tra il 1948 ed il 1993 in Francia, Germania ed Italia, e della loro comparazione con quelle poste tra il 1935 ed il 1985 negli Stati Uniti (Shapiro e Page, 1988), Isernia, Juhasz e Rattinger (1998) hanno trovato che non più del $56 \%$ di tutte le domande, chieste con identica formulazione per almeno due volte, mostrano

7 Precedentemente, la comunicazione politica era essenzialmente mediata dalle realtà subculturali, sebbene già alla metà degli anni '60, rileva Sani (1974), la presa dei media partitici sul pubblico era scarsa. 
cambiamenti nelle risposte superiori a 6 punti percentuali. Questo ammontare di cambiamento è molto simile a quello degli altri paesi. A conferma, Shapiro e Page $(1988,217)$ calcolano che, negli Stati Uniti, in politica estera solo il $58 \%$ dei cambiamenti significativi era improvviso ${ }^{8}$. I relativi valori per l'Italia sono molto simili, ammontando al $64 \%$ dei casi.

La diffusa convinzione tra decisori, opinionisti e osservatori che l'opinione pubblica italiana in politica estera sia altamente volatile e difficile da prevedere non sembra quindi corroborata dai dati disponibili. L'opinione pubblica italiana non cambia atteggiamento improvvisamente o più frequentemente del pubblico americano, tedesco o francese (Oreglia 1999). Non solo, ma come si è cercato di mostrare altrove (Battistelli e Isernia 1992; Isernia 1996b; Bellucci 1998; Isernia, Juhasz e Rattinger 1998; Bellucci e Isernia, in stampa), a livello aggregato l'opinione pubblica cambia in maniera «ragionevole», reagendo in maniera significativa e realistica sia agli eventi internazionali che ai mutamenti nei rapporti di forze Est-Ovest.

L'impatto dell'opinione pubblica sulla politica estera italiana. Se quindi l'opinione pubblica italiana non appare radicalmente differente da quella di altri paesi, si potrebbe comunque sostenere che il suo impatto sulle politiche sia differente, per la diversa natura del sistema istituzionale e del ruolo che in esso gioca l'opinione pubblica di massa. In altre parole, come alcuni sostengono, a prescindere da quali siano gli orientamenti del pubblico e da come essi varino nel tempo, essi sono irrilevanti per spiegare i cambiamenti di policy dei governi italiani.

Un esame comparato rileva tuttavia che, nonostante crescenti evidenze empiriche mostrino come opinione pubblica e politiche siano collegate (Shapiro e Jacobs 1989; Jacobs e Shapiro, 1994), in realtà non siamo ancora in grado di rispondere con precisione a domande cruciali sulle condizioni e i processi attraverso i quali l'opinione pubblica incide sulle politiche. I tradizionali modelli di rapporto élite-pubblico del tipo topdown e bottom-up sono non solo descrittivamente inadeguati a

8 Page e Shapiro $(1982,28-29)$ hanno classificato i casi di cambiamento significativo (superiore cioè a 6 punti percentuali) in graduali, improvvisi e fluttuazioni. Un cambiamento è improvviso «se avviene ad un tasso del $10 \%$ o più all'anno» ed una fluttuazione «è definita dal numero di cambiamenti di direzione dei cambiamenti significativi entro un dato intervallo di tempo». 
dar conto della complessa natura del rapporto tra opinioni di massa e politiche, ma anche teoricamente incapaci di cogliere la natura essenzialmente «dinamica» di questa interazione. L'impatto del pubblico sulle politiche non è infatti un fenomeno statico, del tipo c'è-o-non-c'è, ma un processo influenzato da molti fattori, di cui non solo il peso, ma anche il ruolo, la natura, cambia nel corso del tempo.

Non disponendo ancora in Italia di ricerche sistematiche sui rapporti tra pubblico e politiche ${ }^{9}$, ci limiteremo ad avanzare quattro osservazioni generali sul rapporto tra opinione pubblica e politiche, per poi concentrarci sulla politica estera.

La prima osservazione è che nell'Italia del dopoguerra si è consolidata una rigida separazione tra circuito elettorale e circuito delle opinioni, con una netta prevalenza del primo sul secondo, tanto da portare ad una atrofizzazione del secondo. Come ha rilevato Cartocci $(1990,148$ e ss.) vi è «un'indipendenza quasi sistematica fra le opzioni di voto e le opinioni espresse, vale a dire fra comportamenti e atteggiamenti», per cui lo «spazio elettorale», definito dai comportamenti di voto, e lo «spazio politico», che consiste negli atteggiamenti nei confronti della politica, non coincidono né sono collegati.

La seconda osservazione attiene al ruolo, nella connessione opinion-policy, che assumono i partiti politici. In generale, l'importanza dei partiti politici come canale di comunicazione delle preferenze del pubblico al sistema politico è talmente ovvia nel caso italiano da non dover essere rimarcata ulteriormente. In una società divisa in subculture forti e ben radicate, che dividono verticalmente l'elettorato, in cui i canali di comunicazione politica sono plasmati prevalentemente dai partiti politici ed in cui i mass media sono fortemente controllati e comunque influenzati da gruppi politici od economici, l'immagine di una opinione pubblica in mutamento, suscettibile di cambiamenti si fa strada con difficoltà. D'altra parte, l'estrema stabilità dell'elettorato rendeva in Italia meno necessario apprestare strumenti di raccolta di informazioni diretti ad accertare le opinioni del pubblico alternative alle elezioni stesse. Tuttavia, dal momento che alle elezioni non si può chiedere molto di più di

9 Per la verità, nemmeno molte sulle opinioni del pubblico su specifiche policies (un'eccezione Guidorossi, 1984). Un qualche interesse per il ruolo dei sondaggi in politica comincia ad emergere a seguito dell'ingresso di Berlusconi in politica. Su questo tema una recente raccolta di contributi è in Ceri (1997). 
quello che possono dare - il voto è una forma di partecipazione «strumentale» a basso contenuto specifico (in termini di preferenze di policy), soprattutto nei sistemi multipartitici - ecco la sorpresa con cui la classe politica ha reagito nei pochi momenti nei quali l'opinione pubblica è stata chiamata ad esprimere le sue preferenze, come nei referendum a più elevato contenuto di opinione (sul divorzio e sull'aborto).

Un terzo ordine di considerazioni è di natura culturale, e si affianca alle ragioni istituzionali quale spiegazione della scarsa legittimità delle inchieste di opinione. Non è solo il fatto che la scienza politica italiana, nella tradizione elitista alla Mosca ha concentrato la sua attenzione soprattutto sui partiti (Panebianco 1989), quanto piuttosto che la cultura politica di un popolo incide sugli strumenti di raccolta di informazione di cui i decisori si dotano e che ritengono legittimi per capire gli orientamenti della popolazione. L'uso delle inchieste di opinione come strumento politico presuppone alcune pre-condizioni culturali per affermarsi. Una prima condizione - della cui rilevanza politica troviamo traccia nell'immediato dopoguerra in un dibattito che contrappose in America Blumer (1953) a Lazarsfeld (1957) e Berelson (1952) (si veda anche Converse 1987) - è l'esistenza di una concezione «individualista» della società e dell'opinione pubblica. In effetti, tra i sociologi e i decisori europei, è proprio la concezione «sociologica» a essere prevalente, con tutte le conseguenze negative (l'insofferenza per le inchieste di opinione) e positive (una concezione più ricca e diversificata della natura dell'opinione pubblica e del suo impatto nelle politiche pubbliche) che ciò comporta (Price 1992). Inoltre, nella cultura politica e accademica italiana, imbevuta di storicismo ed idealismo, l'opinione pubblica come somma non ponderata di opinioni individuali non ha una sua legittimità autonoma (Battistelli 1996). Insomma, l'atteggiamento empirico e positivista che soggiace all'idea che «gli atteggiamenti possono essere misurati» ha sempre avuto vita dura in Italia ${ }^{10}$.

10 Questa è forse una delle ragioni per cui le inchieste di opinioni siano arrivate nello stock and trade degli scienziati sociali italiani solo con ritardo e siano state accettate con riluttanza. Per una interessante analisi delle reazioni italiane allo studio sulla «cultura civica» negli anni '60 e '70 si veda Sani (1980). Sarebbe interessante ricostruire in che modo la diffusione dei sondaggi tramite il marketing economico abbia contribuito a plasmare il mercato delle inchieste di opinione in Italia e l'immagine di queste presso politici e giornalisti. Per una ricostruzione della nascita di questa industria in America il classico è Converse (1987). 
Un ultimo elemento che contribuisce a questa atrofizzazione è il ruolo dei media in Italia. Rispetto al modello «orientato al mercato economico» (Entman 1989) prevalente in altri paesi, quello italiano potrebbe essere definito come «orientato al mercato politico» (Castronovo e Tranfaglia 1995; Murialdi 1998). Nel primo modello, gli imperativi commerciali delle imprese giornalistiche e televisive guidano sia la ricerca delle notizie che la loro produzione. Vi è perciò un particolare slant delle notizie a favore delle fonti pubbliche ma anche una costante attenzione alle conseguenze di mercato delle notizie prodotte e queste due esigenze, spesso confliggendo tra loro, producono la dinamica conflittuale/consensuale che caratterizza la stampa anglosassone. In un mercato dominato da considerazioni politiche, in cui le imprese giornalistiche o televisive sono di fatto di proprietà pubblica o dipendono fortemente dai contributi finanziari di gruppi imprenditoriali con diversificati interessi economici, le pressioni sui giornalisti e le dinamiche di produzione delle notizie sono differenti. In particolare, il principale compito dei media, almeno di quelli a stampa sino a pochi anni fa, non è visto tanto nel fornire un collegamento tra opinione pubblica e policymakers, quanto un collegamento tra policymakers e gruppi di influenza differenti. È insomma un canale di comunicazione orizzontale tra le élites, invece che tra queste e la massa dei lettori. In questa situazione i politici non solo hanno una più elevata probabilità di vedere le loro storie riportate sulla stampa, ma sono in grado di influenzare il modo stesso di definire il tema oggetto di attenzione da parte dei media e di calibrarne l'impatto sui media stessi. Ciò crea un «effetto specchio» presso i politici i quali utilizzano i media come «surrogato» di misurazioni più precise dell'opinione pubblica, finendo sostanzialmente per guardarsi allo specchio, in un processo di continua «profezia che si autoadempie» che aumenta il rischio di errate letture del clima di opinione.

In questo contesto strutturale, il fatto che, in Italia, la politica estera sia stata per moltissimi anni un settore ad elevatissimo contenuto «simbolico» ha reso possibile ai partiti una formidabile stabilizzazione dell'elettorato lungo l'asse sinistra-destra, con vantaggi per tutti. Una elevata polarizzazione ovviamente costituiva un vantaggio per la Dc che, agitando lo spettro comunista, poteva a piacimento distrarre l'attenzione del pubblico, soprattutto in fase elettorale, dai temi di politica interna 
quando questi non l'avvantaggiavano ${ }^{11}$. Come ha ricordato Pizzorno $(1980,99-154)$, anche al Pci la politica estera ha fornito per decenni (sostanzialmente sino ai primi anni '70) contenuti di mobilitazione su temi «alti» in una lunga fase in cui questo partito, per la propria strategia politica interna, aveva interesse ad attenuare e temperare le spinte rivendicative provenienti dal sindacato. La politica estera insomma è stato un utile tema con cui il Pci ha potuto imporre al sindacato il rinvio della lotta di classe allo scopo di consentire al partito stesso di sopravvivere e, sperabilmente, di crescere elettoralmente.

$\mathrm{Ne}$ consegue che quando si trattava di fare delle scelte concrete (e scelte di questo tipo ve ne sono state diverse nei decenni postbellici) l'unica soluzione per il governo era quella di prenderle in assoluto segreto, al riparo dagli occhi e dalle orecchie dei partiti, con la conseguenza di frapporre un «velo di ignoranza» tra $\mathrm{i}$ decisori e l'opinione pubblica, in linea con il dettato realista che vede nell'opinione pubblica una guida troppo instabile delle scelte dei governanti.

In questa situazione, il crollo del blocco sovietico e la crisi del sistema dei partiti ristrutturano il quadro nel quale l'interazione opinione-politiche avviene. Tre sono i cambiamenti significativi che ci preme sottolineare. Il primo è la radicale depolarizzazione della frattura Est-Ovest nella politica italiana. Sebbene il lento movimento dei partiti di sinistra verso il riçonoscimento delle alleanze politico-militari del nostro paese fosse cominciato praticamente già alla metà degli anni ' 50 , è solo con il crollo del Muro di Berlino che le scelte di sicurezza del nostro paese - in particolare il sostegno per la Nato - hanno acquisito un reale consenso trasversale da sinistra a destra ${ }^{12}$.

Questo mutato atteggiamento verso la Nato e le scelte di sicurezza italiane si accompagna ad un secondo importante cam-

11 Al fallimento della strategia di giocare la carta esterna per minimizzare le difficoltà di politica interna Putnam $(1977 ; 1978)$ attribuisce la minore presa della campagna anticomunista della Dc nelle elezioni del 1976.

12 Può essere interessante segnalare, a testimonianza della persistenza di un latente anti-americanismo in alcuni settori dell'elettorato di sinistra, come tra gli elettori comunisti (poi Pds) la percentuale di intervistati che desiderano che gli europei abbiano una maggior voce in capitolo nell'organizzazione militare sia la più alta (Bellucci 1998). A questo atteggiamento soggiace apparentemente la tendenza a sottolineare il ruolo politi$c o$ della Nato piuttosto che quello militare, e una maggiore influenza europea è ritenuta una condizione perché le considerazioni politiche figurino come preminenti rispetto a quelle militari (Isernia 1996b). 
biamento di atteggiamento, quello nei confronti delle nostre forze armate, che per più di quarant'anni erano state una presenza «invisibile» (Battistelli 1996) nella nostra società. A partire dagli anni ' 80 , ed in particolare con la missione multinazionale in Libano agli inizi del decennio e per il ruolo giocato dall'esercito nel soccorso alle popolazioni colpite dalle catastrofi naturali nel nostro paese, questo atteggiamento è cominciato a cambiare. La fine della guerra fredda ha contribuito ad accelerare questo processo, ed oggi le forze armate sono una istituzione che, se non raggiunge $\mathrm{i}$ livelli di fiducia e di consenso dei Carabinieri, gode di una popolarità di gran lunga superiore rispetto ad altre istituzioni statali e ai partiti, e di un consenso ampio non solo per compiti civili ma anche militari (come la partecipazione a missioni di pace ed a missioni militari in ambito Nato).

Infine, una terza novità è la mutata relazione tra opinione pubblica e politiche governative, almeno per quanto concerne la politica estera. Con la fine della guerra fredda ed il venir meno dell'anticomunismo come collante ideologico della coalizione di governo, il rapporto tra opinione pubblica e politica estera dell'Italia è divenuto più «fisiologico». Una manifestazione di questo mutato rapporto è nella disponibilità del pubblico italiano a sostenere un ruolo più attivo del nostro paese negli affari internazionali e nei fattori che ne spiegano la variazione nel tempo. L'internazionalismo degli italiani - in genere piuttosto alto - risente molto della stabilità dei governi della repubblica. Tra il 1992 ed il 1997, il grado di internazionalismo degli italiani ha conosciuto vari alti e bassi, secondo un andamento che riflette il grado di turbolenza della vita politica del nostro paese ed in particolare l'aspettativa di vita dei governi. Quando un governo è debole, la sua aspettativa di vita piuttosto breve e gravi incertezze circondano la sua capacità di pilotare il paese, il livello di internazionalismo degli italiani diminuisce, mentre aumenta la percentuale di rispondenti che ritiene sia più importante concentrarsi sugli affari interni. Quando un governo gode invece di una ampia maggioranza e la sua aspettativa di vita è medio-lunga, nessuna nube appare all'orizzonte a minacciarne la vita e il livello di internazionalismo aumenta. In altre parole, per gli italiani una efficace politica estera dipende da un governo autorevole e stabile (Bellucci 1998).

Questo è il contesto politico-istituzionale in cui il processo decisionale relativo alla Bosnia ha avuto luogo. 


\section{L'opinione pubblica italiana nella crisi di Bosnia}

La crisi jugoslava scoppia in un momento particolarmente delicato per il sistema politico italiano, accentuando così le normalmente già complesse relazioni di reciproca interdipendenza tra il livello nazionale e quello internazionale. Il collasso della Jugoslavia e la guerra di Bosnia si sono infatti dipanati in concomitanza con il configurarsi di una duplice sfida per l'Italia, sia a livello domestico che a quello internazionale. Da una parte, sul piano interno, a partire dal 1992 il sistema partitico italiano entrava nella più profonda crisi dal dopoguerra. Contemporaneamente, tuttavia, l'Italia si trovava anche ad affrontare una crescente domanda proveniente dal fino ad allora «tranquillo» ambiente internazionale, dove la fine della Guerra fredda sollecitava stati con rango di media potenza - come l'Italia - ad una maggiore presenza sulla scena internazionale. Il collasso della Jugoslavia richiedeva quindi all'Italia una ulteriore e costante presenza nelle sedi internazionali, mettendo sotto pressione le già scarse risorse della classe politica e diplomatica, e del proprio sistema di sicurezza.

Per di più, la dinamica stessa della crisi jugoslava ha contribuito a restringere la capacità dell'Italia di influenzare la politica dell'area ed il corso degli eventi. Dopo una prima fase di marcato attivismo durata fino al 1991, la politica estera italiana nei Balcani è quindi divenuta progressivamente reattiva e di basso profilo (Holmes 1993) ${ }^{13}$. Anzitutto il fallimento dell'azione dell'Unione europea - principale, se non unica, arena ove l'Italia poteva con qualche probabilità di successo promuovere $\mathrm{i}$ propri interessi nazionali - ha grandemente contribuito a deprimere lo spazio di manovra italiano. Le capacità nazionali di influenzare gli eventi sono poi state ulteriormente compresse dal crescente ruolo ricoperto, a causa dell'inefficacia dell'azione europea, dalla diplomazia multilaterale informale guidata dagli Stati Uniti e, alla fine, dal ricorso alle armi. Esclusa dal Gruppo

13 Holmes $(1993,186)$ cita la Bosnia come una interessante eccezione rispetto alla tradizionale irrilevanza dell'opinione pubblica italiana in politica estera. Egli argomenta come l'opinione pubblica italiana sia stata capace di influenzare la politica estera, per di più in una direzione nettamente più interventista rispetto al tradizionale pacifismo nazionale. Come si vedrà nel prossimo paragrafo, questa influenza dell'opinione pubblica, se era vera durante le prime fasi della crisi bosniaca, non durerà, mentre l'opinione interventista rimarrà stabile sino alla conclusione della crisi. 
di contatto sino all'inizio del 1996, incerta se contribuire direttamente all'azione militare alla quale già dava un contributo logistico offrendo delle basi aeree, l'Italia scelse quindi un basso profilo, caratterizzando il suo contributo alla crisi bosniaca sul piano degli aiuti umanitari ${ }^{14}$. In questo contesto, la decisione a fine 1995 di partecipare alla missione dell'Ifor conclude una politica balcanica che ha attraversato due fasi caratterizzate da differenti stili di policy (per un approfondimento, Bellucci, 1998).

Nella prima, che arriva sino al marzo 1994, i governi che si succedettero (Andreotti, Amato, Ciampi) adottarono nei confronti della ex-Jugoslavia una politica di solidarietà e gradualismo negli impegni, ma sostanzialmente di basso profilo. Con la creazione del Gruppo di contatto, la politica italiana verso la Bosnia cambiò, almeno simbolicamente, atteggiamento. Ciò avvenne con il governo Berlusconi, più assertivo anche se continuò a scontrarsi con la riluttanza ad un effettivo coinvolgimento in Bosnia. Questa posizione contraddittoria - chiedere di più senza essere disponibile a sopportarne conseguenze e costi - caratterizzò anche il successivo governo Dini, sino a quando, con gli accordi di Dayton e la decisione di inviare una brigata a $\mathrm{Sa}$ rajevo sotto il comando tattico francese la posizione e la visibilità italiana nella crisi di Bosnia cambiarono e si rafforzarono.

Una analisi al livello aggregato. In questo paragrafo analizzeremo l'andamento degli orientamenti dell'opinione pubblica nazionale nei confronti di un intervento armato in Bosnia durante il periodo 1992-1996, valutando prima l'entità effettiva di tale sostegno e poi le sue determinanti. I dati considerati provengono da una pluralità di fonti, sia italiane che straniere. Si tratta di inchieste demoscopiche condotte, se non altrimenti specificato, attraverso interviste faccia a faccia o telefoniche su campioni rappresentativi della popolazione adulta (dai 18 anni) italiana. Dobbiamo specificare che, sebbene la disponibilità di dati si estenda dall'autunno 1992, solo a partire dal 1993 compaiono domande che affrontano direttamente il tema di un possibile intervento italiano.

I dati disponibili indicano che gli italiani, così come l'opi-

14 Questo ruolo è continuato anche dopo la fine della guerra. Con 50 milioni di dollari, l'Italia è insieme all'Olanda il principale contributore europeo alla ricostruzione della Bosnia. Cfr. «Corriere della Sera», 14 aprile 1996. 
nione pubblica europea (Sobel 1996), hanno sempre fortemente sostenuto un maggiore impegno nella crisi bosniaca e hanno sempre espresso una forte insoddisfazione per l'impegno della comunità internazionale. Nel novembre 1992, il $67 \%$ degli italiani riteneva che sia la Comunità europea sia gli Stati Uniti stessero facendo troppo poco per fermare la guerra in Jugoslavia. Due anni dopo, nel giugno 1994, la percentuale saliva rispettivamente al 70\% (l'Europa) ed al 72\% (gli Usa) mentre solo il $57 \%$ condivideva un analogo giudizio per l'Italia. Queste valutazioni critiche dell'opinione pubblica nazionale sull'impegno della comunità internazionale dipendono, da una parte, dalla convinzione che le organizzazioni internazionali possono esercitare una reale influenza sulla situazione della ex-Jugoslavia e, dall'altra, dal desiderio di vedere ristabilita la pace nell'area. Nel febbraio 1994, infatti, il $74 \%$ degli italiani intervistati dalla Doxa pensava che se la diplomazia internazionale avesse compiuto uno sforzo reale avrebbe avuto molte possibilità di fermare la guerra in Bosnia. Nel maggio dello stesso anno, richiesti di identificare chi avesse effettive capacità di risolvere il conflitto nella ex-Jugoslavia, la maggioranza dell'opinione pubblica italiana metteva al primo posto insieme le Nazioni unite, l'Unione europea e la Nato. Solo un singolo paese raggiungeva una posizione comparabile: gli Stati Uniti, indicati dal 55\% degli intervistati come capaci di svolgere un ruolo importante nella risoluzione della crisi.

Due domande, con una formulazione solo leggermente differente, poste in due sondaggi Doxa del 31 gennaio e 9 febbraio 1994 - cioè immediatamente prima e dopo il bombardamento del mercato di Sarajevo del 5 febbraio, che fece almeno 68 vittime e 200 feriti - consentono di esaminare l'impatto di un incremento del livello di violenza sull'orientamento dell'opinione pubblica. Il bombardamento ha incrementato di circa 6 punti - dal $51 \%$ al $57 \%$ - la quota di popolazione italiana disponibile a sostenere un intervento armato delle truppe dell'Onu per fermare i combattimenti in Bosnia. Va segnalato come l'impatto del bombardamento sia soprattutto sugli incerti piuttosto che sulla quota di opinione pubblica contraria ad un intervento armato, mentre l'effetto delle uccisioni indiscriminate sul sostegno dell'opinione pubblica per un intervento militare italiano è molto più esteso tra coloro che erano già a favore di un intervento Onu. Nel sondaggio del 31 gennaio il $73 \%$ degli italiani favorevoli ad un intervento dell'Onu sosteneva anche la parteci- 
pazione militare italiana. Quattro giorni dopo l'attacco al mercato di Sarajevo, il sostegno salì all' $85 \%$, segnalando come chi sosteneva un intervento militare condivideva l'opinione che l'Italia dovesse farne parte.

Sin dal marzo 1993 il pubblico italiano era favorevole ad un intervento militare delle Nazioni unite ed entro la fine del 1994 questa convinzione appariva ampliata e rafforzata. Un sondaggio del novembre 1994 segnalava come il 54\% degli intervistati riteneva che la guerra in Bosnia dovesse essere fermata ad ogni costo entro la primavera, mentre il $68 \%$ riteneva che le truppe dell'Onu non dovessero essere ritirate anche a fronte di un inasprimento dei combattimenti. Tale sostegno aumenta, salendo al $77 \%$ nel dicembre 1994 , se la domanda menziona esplicitamente che le truppe sono inviate per mettere in opera un accordo di pace. Questo livello è analogo al risultato (74\%) emerso in un sondaggio del novembre 1993, in risposta ad una doman$\mathrm{da}$ che non faceva esplicito riferimento a un accordo di pace ma solo a un accordo tra le parti in conflitto.

Se esaminiamo l'evoluzione dell'opinione pubblica riguardo l'intervento militare italiano si conferma la sostanziale stabilità nel sostegno a un intervento delle nostre forze armate (fig. 1), contraddicendo quindi l'immagine che delle preferenze dell'opinione pubblica avevano le élites politiche. L'unico cambiamento accade nella metà del 1995 , quando la critica posizione dei caschi blu ostaggio dei serbi e l'evidente incapacità delle Nazioni unite ad arrestare gli attacchi a Sarajevo fecero cadere il livello del sostegno per un intervento armato.

Dobbiamo tuttavia chiederci quanto stabile fosse questo sostegno, dal momento che, come abbiamo già visto, nel 1994 l'interpretazione di alcuni politici metteva l'accento sulla fragilità del sostegno e sull'alta probabilità di un suo dissolvimento non appena si fosse incorsi in perdite di vite umane tra i militari della forza multinazionale. Si tratta di un tema importante e delicato, che chiama in causa la disponibilità dell'opinione pubblica a sopportare i costi conseguenti alle scelte preferite (Everts e Isernia in stampa). E stato infatti argomentato che le nazioni occidentali sono progressivamente più riluttanti ad accettare perdite di vite umane tra le proprie truppe per interventi militari all'estero (Luttwak 1994). Nel caso italiano - un paese cioè che non ha (fortunatamente) combattuto alcuna guerra dal 1945 e la cui opinione pubblica appare sistematicamente la meno disponibile, a livello comparato, a fare sacrifici per il pro- 


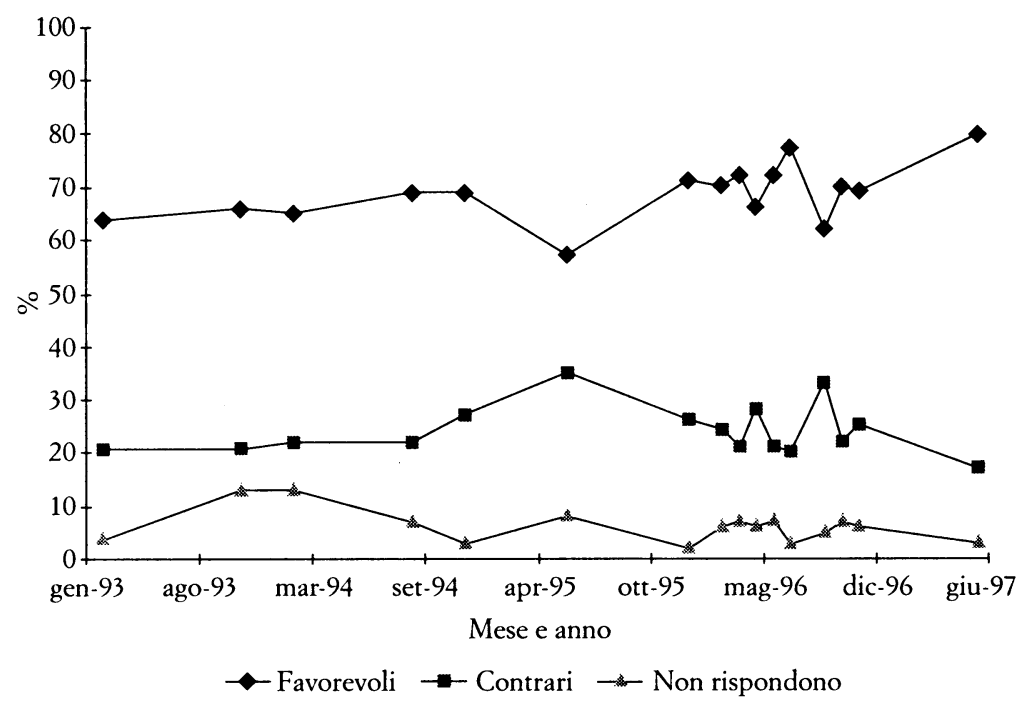

FIG. 1. Evoluzione del sostegno per l'intervento militare italiano in Bosnia.

prio paese - questa tesi sembra particolarmente persuasiva. In effetti, la figura 2 mostra come il sostegno per un intervento militare italiano si contragga all'aumentare del livello di sacrificio richiesto. L'andamento tuttavia appare simile a quello riscontrato in altri paesi (Everts 1996) e mostra come il livello di sostegno sia reso significativamente più fragile da una serie di condizioni progressivamente più sfavorevoli, passando cioè dalla sola minaccia all'effettivo uso della forza, dalla probabilità di incorrere in perdite tra $i$ nostri soldati e tra quelli nemici, fino alla eventualità della perdita di parenti o di un coinvolgimento in prima persona nei combattimenti. Questo risultato, che mostra un evidente trend negativo, deve essere tuttavia opportunamente inserito nel contesto appropriato. Il sostegno per un intervento nonostante perdite di vite umane non è correlato esclusivamente con il livello del coinvolgimento personale (come nella domanda esaminata), ma anche a fattori quali la chiarezza e la legittimità degli obiettivi perseguiti, il senso di appartenenza ad una forza multinazionale, la comprensione che la sopportazione è temporalmente limitata, la durata del conflitto, e così via. L'esperienza della guerra del Golfo e della Somalia, dove undici soldati italiani (più 4 civili) furono uccisi e ancor 


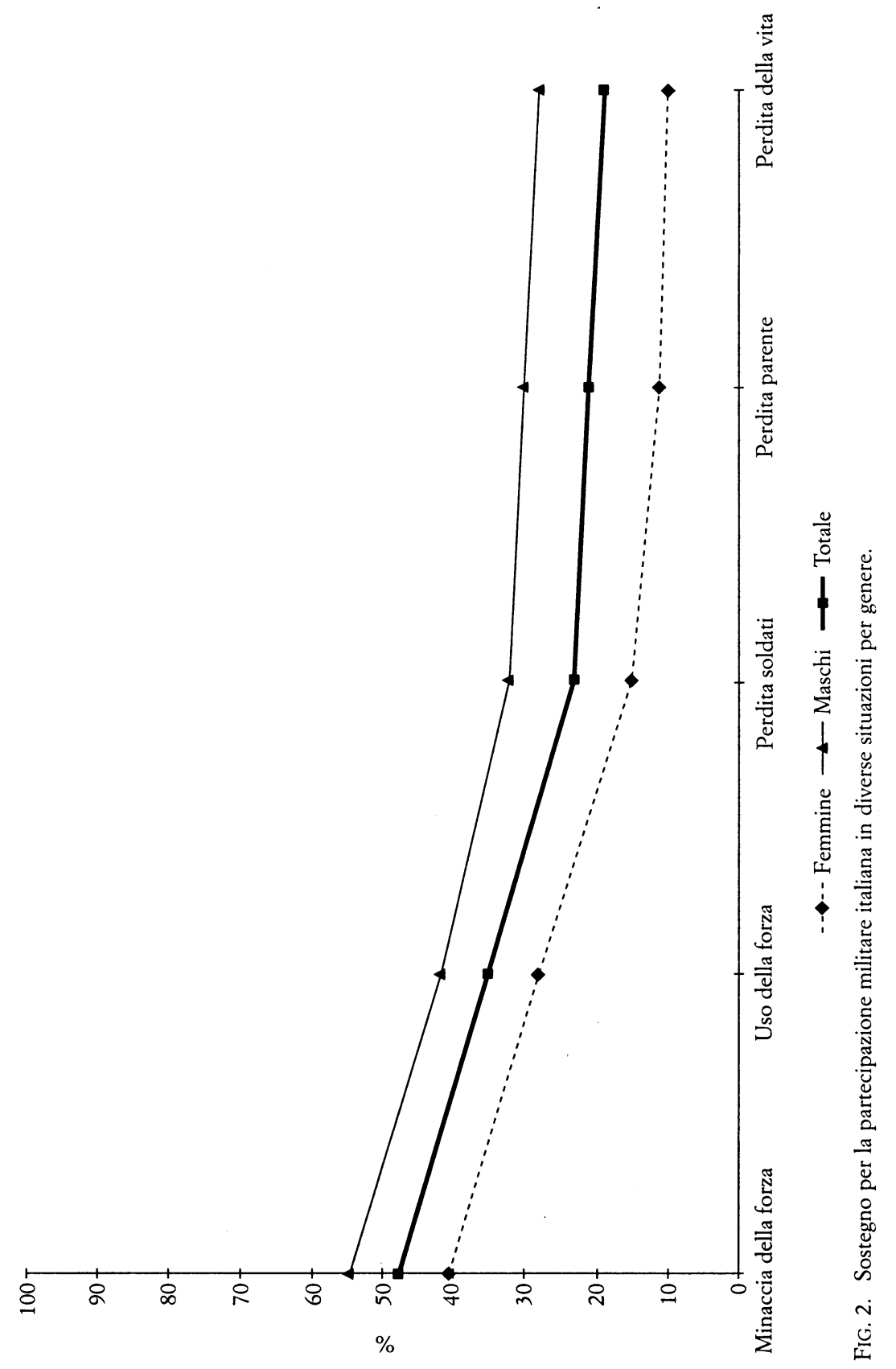


più feriti, segnala infatti che quando gli obiettivi sono chiari, la legittimità dell'operazione militare è ben compresa e l'intervento è temporalmente limitato, l'opinione pubblica italiana è nel complesso disponibile a sostenere alcuni costi umani connessi all'operazione militare.

Le determinanti del sostegno a livello individuale. Passando da una analisi aggregata ad una al livello individuale, cercheremo ora di delineare i fattori correlati con l'opinione sulla partecipazione italiana alla missione militare in Bosnia, verificando anche eventuali cambiamenti nella direzione di questi effetti nel tempo. Prenderemo in esame due differenti basi di dati. La prima è un monitoraggio mensile dell'opinione pubblica, dall'avvio della missione Ifor nel gennaio 1996 sino al novembre dello stesso anno. Esso consente una accurata analisi dell'evoluzione dell'orientamento degli italiani nell'arco di un anno, pur presentando un limite connesso al ristretto numero di variabili di tipo motivazionale incluse nei sondaggi. Per superare tale limitazione prendiamo anche in esame una seconda fonte, Difebarometro n. $4^{15}$, un sondaggio che abbiamo condotto nel luglio 1996 il quale presenta un più ampio spettro di variabili motivazionali e di opinione.

Il nostro livello di conoscenza sulla reattività dell'opinione pubblica, a livello individuale, alle crisi internazionali che coinvolgono direttamente l'Italia è meno ampio ed approfondito della nostra comprensione della generale evoluzione dell'opinione pubblica in politica estera al livello aggregato. In effetti, gli unici studi disponibili si circoscrivono alla guerra del Golfo (Ammendola 1993; Isernia 1996b). Diversamente da altri paesi, come Francia e Stati Uniti, in Italia il consenso per l'intervento italiano nel Golfo è divenuto maggioritario solo settimane dopo lo scoppio delle ostilità, una volta cioè che la guerra apparve essere breve, con poche perdite (per gli occidentali) e senza rischi di escalation a livello sia regionale (Israele) che globale (Urss). Il ruolo dei media emerse comunque importante nel veicolare l'immagine di una guerra vincibile e «pulita» e di un apparato militare internazionale efficace. I risultati dei sondaggi all'epoca svolti indicano che la più forte opposizione alla guerra proveni-

15 Difebarometro è un programma di inchieste campionarie periodiche su temi di politica estera e della sicurezza realizzato a partire dal 1994 dall'Archivio Disarmo di Roma e da Swg di Trieste. 
va dalle donne, dalle generazioni più anziane, da coloro con un basso livello d'istruzione e da coloro politicamente orientati a sinistra. $\grave{E}$ interessante comparare questi risultati con quelli relativi alla crisi della Bosnia, per valutare somiglianze e differenze.

Per questo abbiamo condotto una analisi di regressione logistica ove abbiamo anche inserito l'interazione tra ciascuna variabile ed il mese del sondaggio, in modo da modellare l'influenza della variabile nel tempo. I risultati, riportati nella tabella 1 , indicano come le principali variabili esplicative del consenso per la missione dell'Ifor - cioè gli effetti diretti netti - siano la generazione, l'occupazione, la scelta partitica e l'orientamento internazionale. Per quanto riguarda il fattore tempo, solo l'età, l'occupazione e la preferenza partitica mostrano un mutamento della direzione di impatto nel periodo considerato. Tra i più giovani (18-24 anni) la probabilità di sostenere la missione è cinque volte più grande rispetto ai più anziani ${ }^{16}$. Probabilmente la socializzazione durante la Seconda guerra mondiale (per la generazione precedente al 1945) e durante la Guerra fredda (la generazione successiva) deprime il sostegno per le missioni di tipo militare ${ }^{17}$. Tuttavia sono i giovani che mostrano, col procedere della missione, una leggera tendenza alla contrazione del sostegno. Considerando la struttura occupazionale, gli impiegati e le casalinghe hanno la più alta probabilità di esprimere una valutazione positiva sulla missione in Bosnia. Entrambi questi gruppi, tuttavia, presentano un andamento negativo nel tempo. Le casalinghe hanno così una probabilità media 4,6 volte superiore rispetto ai lavoratori autonomi di essere a favore dell'intervento italiano, ma il loro sostegno diminuisce mensilmente del $13,1 \%$. Anche gli impiegati hanno una più alta probabilità di essere favorevoli (pari a 1,8 la probabilità degli autonomi), ma ogni ulteriore mese di svolgimento della missione deprime il loro consenso dell' $11,3 \%$. Una netta differenza emerge quindi tra l'elettorato dei raggruppamenti politici. Tra gli elettori dell'Ulivo la probabilità di esprimere un giudizio favorevole sulla

16 Calcolando l'antilogaritmo del coefficiente relativo ai giovani si ottiene una stima dell'impatto della variabile in termini di probabilità rispetto al livello di riferimento (gli anziani), esprimibile a sua volta in valori percentuali. Infatti: $[\exp (1,66)-1] \times 100$ $=425,9 \%$.

17 Per maggiori approfondimenti sull'impatto della generazione e della socializzazione politica sull'orientamento dell'opinione pubblica italiana nei confronti della politica di difesa si vedano Bellucci (1996) e Isernia (1996b). 
TAB. 1 Atteggiamento verso l'intervento italiano in Bosnia. Modello di regressione logistica (contrari verso favorevoli). Serie temporale gennaio-novembre 1996 - Stime di massima verosimiglianza $-N=7591$

\begin{tabular}{|c|c|c|}
\hline & Coefficiente & E.s. \\
\hline \multicolumn{3}{|l|}{ Genere } \\
\hline \multicolumn{3}{|l|}{ Maschio (0) } \\
\hline $\begin{array}{l}\text { Femmina } \\
\text { Femmina } \times \text { Ternpo }\end{array}$ & 0,08 & 0,24 \\
\hline $\begin{array}{l}\text { Femmina } \times \text { Tempo } \\
\text { Zona }\end{array}$ & $-0,04$ & 0,03 \\
\hline \multicolumn{3}{|l|}{ Zona } \\
\hline \multicolumn{3}{|l|}{ Nord-ovest (0) } \\
\hline $\begin{array}{l}\text { Nord-est } \\
\text { Centro }\end{array}$ & 0,39 & 0,31 \\
\hline Centro & 0,04 & 0,32 \\
\hline Sud & 0,25 & 0,29 \\
\hline \multicolumn{3}{|l|}{ Zona $\times$ Tempo } \\
\hline Nord-est & $-0,04$ & 0,04 \\
\hline Centro & 0,01 & 0,04 \\
\hline Sud & 0,00 & 0,04 \\
\hline \multicolumn{3}{|l|}{ Età } \\
\hline \multicolumn{3}{|l|}{45 e oltre $(0)$} \\
\hline $25-44$ & $0,49 * * *$ & 0,28 \\
\hline $18-24$ & $1,66^{*}$ & 0,52 \\
\hline \multicolumn{3}{|l|}{ Età $\times$ Tempo } \\
\hline 25.44 & $-0,00$ & 0,03 \\
\hline $18-24$ & $-0,12 * *$ & 0,06 \\
\hline \multicolumn{3}{|l|}{ Titolo di studio } \\
\hline \multicolumn{3}{|l|}{ Fino scuola media $(0)$} \\
\hline Diploma o laurea & 0,16 & 0,25 \\
\hline Diploma o laurea $\times$ Tempo & $-0,00$ & 0,03 \\
\hline \multicolumn{3}{|l|}{ Occupazione } \\
\hline Imprenditore-autonomo & 0,45 & 0,51 \\
\hline Dirigente & 0,86 & 0,55 \\
\hline Impiegato & $1,03 *$ & 0,54 \\
\hline Operaio & 0,40 & 0,50 \\
\hline Pensionato & 0,33 & 0,55 \\
\hline Studente & $-0,31$ & 0,60 \\
\hline Casalinga & $1,42 *$ & 0,54 \\
\hline \multicolumn{3}{|l|}{ Disoccupato $(0)$} \\
\hline \multicolumn{3}{|l|}{ Occupazione $\times$ Tempo } \\
\hline Imprenditore-autonomo & $-0,06$ & 0,06 \\
\hline Dirigente & $-0,09$ & 0,07 \\
\hline Impiegato & $-0,12 * *$ & 0,06 \\
\hline Operaio & $-0,03$ & 0,06 \\
\hline Pensionato & $-0,06$ & 0,07 \\
\hline Studente & 0,02 & 0,07 \\
\hline Casalinga & $-0,14 * *$ & 0,06 \\
\hline \multicolumn{3}{|l|}{ Religione } \\
\hline \multicolumn{3}{|l|}{ Praticante $(0)$} \\
\hline Non praticante & 0,18 & 0,22 \\
\hline Non praticante $\times$ Tempo & $-0,02$ & 0,02 \\
\hline \multicolumn{3}{|l|}{ Voto maggioritario } \\
\hline Polo (0) & & \\
\hline Lega & $-0,59 * *$ & 0,25 \\
\hline Ulivo & $0,57^{*}$ & 0,17 \\
\hline
\end{tabular}


TAB. 1. (segue)

\begin{tabular}{lcc}
$\begin{array}{l}\text { Voto maggioritario } \times \text { Tempo } \\
\text { Lega }\end{array}$ & 0,05 & \\
Ulivo & $-0,04^{* *}$ & 0,03 \\
$\begin{array}{l}\text { Orientamento internazionale } \\
\text { Isolazionista }\end{array}$ & 0,02 \\
Internazionalista & & \\
Internazionalista $\times$ Tempo & $1,02^{* *}$ & 0,23 \\
Tempo & 0,00 & 0,03 \\
Gennaio (0) & & \\
Marzo & $0,60^{* *}$ & 0,23 \\
Giugno & $1,36^{*}$ & 0,43 \\
Luglio & $1,20^{* *}$ & 0,48 \\
Settembre & 0,89 & 0,61 \\
Ottobre & $1,86^{* *}$ & 0,69 \\
Novembre & $1,50^{* *}$ & 0,76 \\
Costante & $-1,01^{* *}$ & 0,53 \\
$\chi^{2}$ & 262,4 & \\
Probabilità & 0,000 & \\
$\%$ casi classificati & 75,9 & \\
\hline
\end{tabular}

$* \mathrm{p}<0,01 ; * * \mathrm{p}<0,05 ; * * \mathrm{p}<0,10$

partecipazione dell'Italia alla missione Ifor è, in media, del $77 \%$, superiore a quella degli elettori del Polo. Infine, considerando l'unica variabile cognitiva presente nel monitoraggio, si conferma che un orientamento internazionalista incrementa sensibilmente, del $177 \%$, la probabilità di essere a favore della partecipazione italiana.

Sulla base di questa analisi logistica multivariata, il genere, l'istruzione e la religiosità non mostrano differenze significative, una volta controllato l'effetto delle altre variabili indipendenti. Per quanto riguarda il genere, è evidente che essere donna, di per sé, non influenza la probabilità di essere favorevole o no rispetto agli uomini. È invece il tipo di relazioni sociali nel quale la persona è inserita a fare la differenza. Relazioni sociali prevalentemente domestiche, come indicato dalla condizione di casalinga, aumentano la probabilità di essere favorevole. Inoltre, il gender gap, che emergeva nel caso della guerra del Golfo, si delinea con maggiore probabilità quando la situazione internazionale implica un effettivo uso della forza. La missione di Bosnia nel corso del 1996 aveva viceversa una immagine tendenzialmente pacifica, e l'uso della forza su ampia scala era una probabilità alquanto remota. Per quanto riguarda l'istruzione, il relativo coefficiente, pur presentando l'atteso segno positivo, non è 
TAB. 2. Atteggiamento verso l'intervento italiano in Bosnia. Modello di regressione logistica (contrari verso favorevoli). Luglio 1996 - Stime di massima verosimiglianza $-N=816$

\begin{tabular}{|c|c|c|}
\hline & Coefficiente & E.s. \\
\hline \multicolumn{3}{|l|}{ Genere } \\
\hline Femmine & 0,45 & 0,36 \\
\hline \multicolumn{3}{|l|}{ Zona } \\
\hline \multicolumn{3}{|l|}{ Nord-ovest (0) } \\
\hline Nord-est & 0,51 & 0,52 \\
\hline Centro & $-0,94 * *$ & 0,48 \\
\hline Sud & $-0,63$ & 0,42 \\
\hline \multicolumn{3}{|l|}{ Dimensione comune } \\
\hline $10-100.000$ abitanti & $-0,11$ & 0,37 \\
\hline$>100.000$ abitanti & $-0,44$ & 0,43 \\
\hline \multicolumn{3}{|l|}{ Età } \\
\hline $18-24$ & 0,75 & 0,67 \\
\hline $25-44$ & $-0,04$ & 0,41 \\
\hline \multicolumn{3}{|l|}{45 e oltre $(0)$} \\
\hline \multicolumn{3}{|l|}{ Titolo di studio } \\
\hline \multicolumn{3}{|l|}{ Fino scuola media $(0)$} \\
\hline Diploma o laurea & 0,25 & 0,35 \\
\hline \multicolumn{3}{|l|}{ Occupazione } \\
\hline \multicolumn{3}{|l|}{ Lavoratore dipendente $(0)$} \\
\hline Imprenditore-autonomo & $-0,80 * *$ & 0,46 \\
\hline Pensionato & $-1,16^{*}$ & 0,55 \\
\hline Casalinga & $-0,65$ & 0,64 \\
\hline Studente & $-0,97$ & 0,67 \\
\hline Disoccupato & $-0,31$ & 0,77 \\
\hline \multicolumn{3}{|l|}{ Religione } \\
\hline \multicolumn{3}{|l|}{ Non praticante $(0)$} \\
\hline Praticante & $-0,07$ & 0,33 \\
\hline \multicolumn{3}{|l|}{ Autocollocazione politica } \\
\hline \multicolumn{3}{|l|}{ Sinistra (0) } \\
\hline Centro-Sinistra & 0,72 & 0,55 \\
\hline Centro & $1,03 * *$ & 0,53 \\
\hline Centro-Destra & $1,53 * *$ & 0,67 \\
\hline Destra & 0,59 & 0,76 \\
\hline \multirow{2}{*}{\multicolumn{3}{|c|}{$\begin{array}{l}\text { Partito votato } \\
\text { Altri }(0)\end{array}$}} \\
\hline & & \\
\hline Ulivo & $1,70^{*}$ & 0,51 \\
\hline Polo & $1,28 * *$ & 0,50 \\
\hline \multicolumn{3}{|l|}{ Valori } \\
\hline \multicolumn{3}{|l|}{ Misti (0) } \\
\hline Materialisti & $-0,66$ & 0,60 \\
\hline Postmaterialisti & $-0,86 * * *$ & 0,57 \\
\hline \multicolumn{3}{|l|}{ Orientamento internazionale } \\
\hline Internazionalista & $1,25^{*}$ & 0,34 \\
\hline \multicolumn{3}{|l|}{$\begin{array}{l}\text { Patria } \\
\text { Palla }\end{array}$} \\
\hline Italia (0) & & \\
\hline Localisti & $-0,32$ & 0,41 \\
\hline Cosmopoliti & $-0,14$ & 0,40 \\
\hline
\end{tabular}


TAB. 2. (segue)

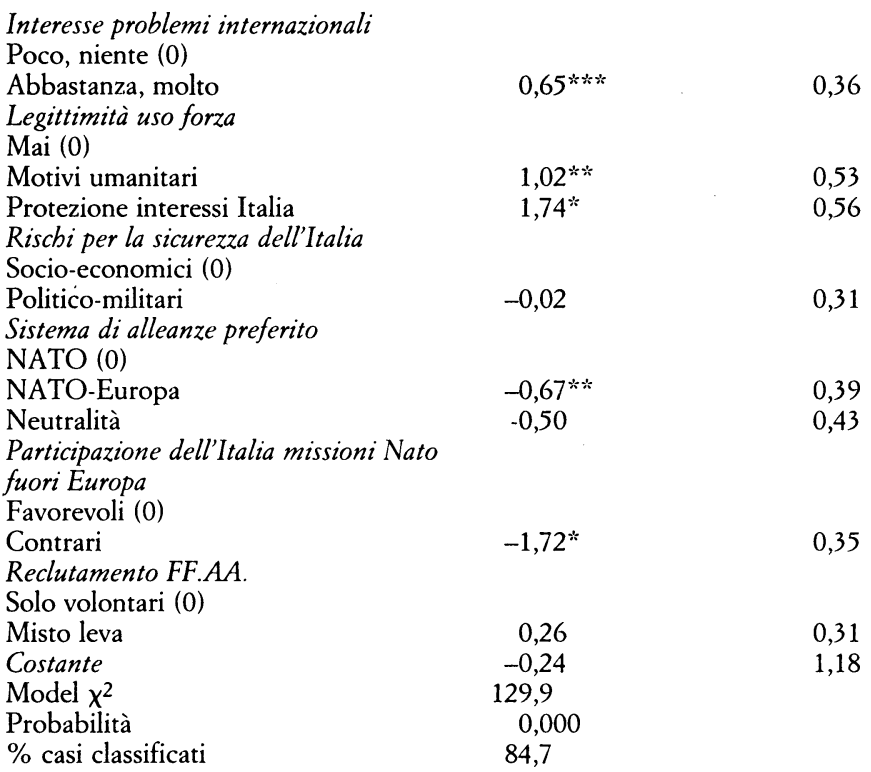

$* \mathrm{p}<0,01 ; * \mathrm{p}<0,05 ; * * \mathrm{p}<0,10$.

significativo. Ciò è dovuto alla sua forte intercorrelazione con la variabile relativa all'orientamento internazionale, che cattura gran parte della sua varianza ${ }^{18}$. Vanno considerati, infine, $i$ coefficienti relativi alla religiosità e alla sua interazione con il tempo. Sebbene non significativi, testimoniando così l'assenza di effetti diretti netti, essi sono nella direzione attesa: in media, $\mathrm{i}$ praticanti sostengono con minore forza la missione rispetto ai non praticanti. Con il tempo, tuttavia, i primi incrementano sistematicamente il loro livello di sostegno.

Passando ora ad approfondire l'analisi del sostegno dell'opinione pubblica italiana per la missione Ifor sulla base dell'indagine Difebarometro 4, realizzata nel luglio 1996, al culmine della ripresa di consenso dopo le elezioni primaverili e che presenta un più ricco spettro di variabili indipendenti, appaiono alcune conferme dei risultati già discussi: l'assenza del gender gap, il

18 Gli isolazionisti sono infatti in generale meno istruiti degli internazionalisti (Isernia 1996b). 
più ampio sostegno dei giovani, dei più istruiti, dei cattolici praticanti, l'influenza dell'orientamento partitico, mediato dalla vicinanza ai partiti che sostengono il governo Prodi. Maggiore interesse presentano le variabili attitudinali, che catturano l'orientamento nei confronti della politica internazionale e la difesa che, come abbiamo già segnalato, costituiscono attualmente le principali fonti delle differenze di opinione in politica estera e di sicurezza. In sostanza, i risultati di un'analisi di regressione logistica (tab. 2) evidenziano, nel complesso, un più ampio impatto degli orientamenti cognitivi rispetto alle variabili socio-demografiche, mentre si conferma l'influenza di quelle politiche. Tra le prime, solo l'occupazione e l'area di residenza esercitano significativi effetti diretti netti: il sostegno declina tra i lavoratori autonomi ed $\mathrm{i}$ pensionati ed è inferiore tra $\mathrm{i}$ residenti nel Mezzogiorno. Tra le seconde, chi si autocolloca al centro-destra dello spazio politico esprime maggior consenso di chi si colloca a sinistra, ma tra gli elettori dell'Ulivo la probabilità di essere favorevole all'intervento è doppia rispetto a quella degli elettori del Polo ${ }^{19}$.

\section{Quali connessioni tra opinioni e politiche?}

I dati discussi nella sezione precedente confermano quanto le evidenze empiriche disponibili su altri paesi mostrano a proposito della Bosnia e che Sobel ha così sintetizzato $(1996,145)$ a livello europeo: «... preferenze relativamente forti della cittadinanza, in particolare per un'azione multilaterale, e politiche dei governi relativamente deboli, perfino sull'impiego delle forze alleate». Anche nel caso italiano, i dati disponibili mostrano, da un lato, l'esistenza di un forte e stabile sostegno per un attivo coinvolgimento delle truppe italiane in Bosnia e, dall'altro, una estrema riluttanza dei governi italiani, di qualsiasi colore politico, a considerare e perfino discutere apertamente di un tale coinvolgimento. Il sostegno pubblico per la partecipazione italiana alla risoluzione della crisi bosniaca si è cristallizzato abbastanza presto nel tempo (all'incirca all'inizio del 1993, per quanto i nostri dati ci consentono di accertare) ed è restato tale

19 Infatti per gli elettori dell'Ulivo questa probabilità è $[\exp (1,7)-1] \times 100=$ $447,4 \%$, mentre per il Polo è $[\exp (1,28)-1] \times 100=259,7 \%$. 
sino a quando le truppe italiane non furono effettivamente schierate, nel quadro dell'accordo di Dayton. Questo sostegno inoltre permane attraverso l'intero spettro di posizioni partitiche, con la parziale eccezione dei comunisti di Rifondazione. L'unico caso di declino nel sostegno che i nostri dati mostrano, quello del luglio 1995, sembra inoltre essere dovuto più all'insoddisfazione per il modo in cui le Nazioni unite svolgevano il loro compito sul terreno piuttosto che alla percezione che i rischi si fossero accresciuti per le truppe occidentali in Bosnia. Nonostante ciò, in tutte le occasioni, e furono diverse come si è visto, in cui il governo italiano fu, direttamente o indirettamente, invitato a considerare la possibilità di inviare le truppe in Bosnia, la decisione ufficiale italiana fu negativa ${ }^{20}$.

Questa disparità tra un pubblico «permissivo» e decisori riluttanti combacia con l'immagine realista del pubblico come volatile ed emotivo e delle élites come realiste e responsabili. Queste conclusioni sono poi in linea con un altro risultato di Sobel (1996, 155): l'orientamento «permissivo» del pubblico «contrasta con l'impressione di molti governi e dei media che il pubblico fosse poco desideroso di interferire con il conflitto in Bosnia». Come abbiamo rilevato all'inizio in almeno un'occasione la decisione italiana di non partecipare fu giustificata esplicitamente con la riluttanza dell'opinione pubblica italiana a sostenere l'uso della forza e le perdite che questa avrebbe potuto comportare, in una riformulazione nostrana della c.d. body-bag bypothesis. In altre parole, il governo italiano basò la sua decisione di non inviare truppe in Bosnia sull'argomento che l'opinione pubblica italiana era in maggioranza contraria a tale intervento. Questo non è propriamente un approccio realista, il quale tende piuttosto ad ignorare l'opinione pubblica se non ne ha bisogno per una più efficace conduzione della politica estera. Visto che i dati empirici disponibili mostrano che, al contrario, l'opinione pubblica italiana era fortemente favorevole, perché il governo italiano usò questi argomenti? Quale «opinione

20 Le decisioni cui facciamo riferimento sono quella del settembre 1992 di allargare l'Unprofor alla Bosnia, quella del gennaio-marzo 1994 sulla richiesta inglese di coinvolgere le truppe italiane, quella del dicembre 1994 su come aiutare le truppe Onu a ritirarsi in caso di bisogno, quella del luglio 1995 di organizzare gli attacchi aerei. In tutti e quattro i casi, il governo italiano decise di non aderire alle richieste di contribuire con truppe. Nel luglio 1995 alla fine il governo concesse l'uso degli aerei italiani per bombardamenti tattici e sostegno a terra (close air support). 
pubblica» aveva in mente? In quest'ultima sezione ritorniamo quindi alla domanda da cui eravamo partiti agli inizi del nostro articolo, muovendoci però dall'esame di un caso isolato all'analisi della relazione tra opinione pubblica e politica nel tempo, a livello aggregato.

A questo scopo, guarderemo alla relazione tra mass media, opinione pubblica e partiti nel periodo compreso tra il 1992 ed il 1996 nel tentativo di capire chi influenzava chi nel processo decisionale concernente la Bosnia. Mancando di dati affidabili e validi sulla natura delle preferenze, abbiamo utilizzato il livello di attenzione come variabile-proxy del grado di coinvolgimento di partiti e mass media nella crisi bosniaca. Per accertare il livello di attenzione dei media ci siamo concentrati sulla stampa, ed in particolare sullo spazio dedicato alla questione bosniaca dal «Corriere della Sera», misurato in numero di articoli mensilmente dedicati alla crisi. Quanto ai partiti, ci siamo concentrati sul momento parlamentare, anche per la sua rilevanza ai fini di qualsiasi decisione in materia. Per misurare il livello di attenzione dedicato alla crisi bosniaca dalle forze politiche in parlamento abbiamo raccolto tutti i dibattiti svoltisi in commissione o in aula dalla Camera dei deputati e contato i giorni (o le porzioni di giorno) dedicati alla discussione della questione bosniaca o alle sue implicazioni per l'Italia. Abbiamo poi comparato questi trends con quello del sostegno all'intervento armato da parte dell'opinione pubblica italiana. Si tratta ovviamente di un approccio grossolano sia per la natura degli indicatori scelti sia per la difficoltà di enucleare la direzione dell'attenzione dei media e dei parlamentari, in altre parole se e quanto media e parlamentari erano favorevoli o contrari all'intervento. Fermo restando quindi che si comparano variabili differenti (il livello di attenzione dei parlamentari e dei media e quello di sostegno del pubblico), riteniamo tuttavia che questi dati ci forniscano un'indicazione sufficientemente precisa della natura delle interazioni tra questi tre gruppi.

Le figure 3 e 4 mostrano i risultati, comparando bilateralmente il livello di attenzione dei media rispettivamente con quello dei parlamentari, espresso come tempo dedicato a questo tema in Parlamento, e con il sostegno del pubblico. Da questi trends emerge abbastanza chiaramente la stretta connessione tra attenzione dei media e dei parlamentari alla Bosnia e la rimarchevole stabilità dell'opinione pubblica, anche in presenza di oscillazioni marcate nello spazio dedicato a questi ar- 


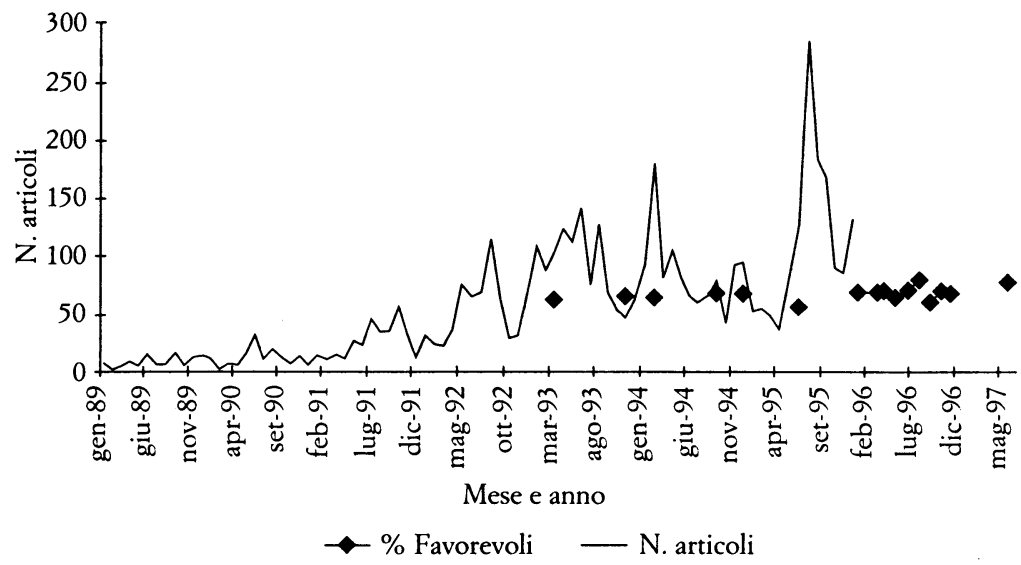

FIG. 3. Evoluzione attenzione dei media e sostegno del pubblico.

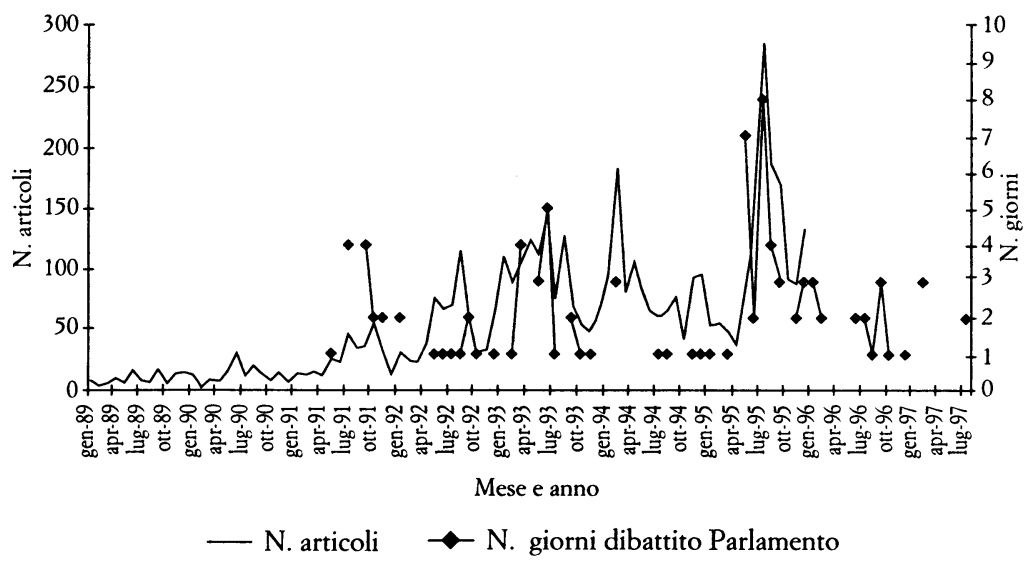

FIG. 4. Evoluzione attenzione dei media e del Parlamento.

gomenti nella stampa quotidiana. L'attenzione dedicata dal «Corriere della Sera» alla Bosnia è da un lato crescente nel corso degli anni, ma con ampie oscillazioni, collegate sostanzialmente alla natura degli sviluppi in Bosnia. Atrocità contro le popolazioni civili, offensive militari ed iniziative di pace spiegano l'andamento altalenante dell'attenzione. Il parlamento non mostra un trend di crescita dell'attenzione, ma le oscillazioni 
sono strettamente collegate a quelle che riscontriamo nei media. Questo significa che media e parlamento si influenzano probabilmente a vicenda ed entrambi sono dominati, nella fissazione della loro agenda, dagli sviluppi in loco. Questi due trends comunque non sono collegati a quelli dell'opinione pubblica, la quale tra l'altro, e questo è senza dubbio il risultato più interessante, non mostra né un trend di crescita del sostegno legato ad una crescente attenzione per questo tema da parte dei media, né oscillazioni collegate agli sviluppi sul terreno. In altre parole, ben lungi dall'essere il pubblico a reagire emotivamente alla situazione in Bosnia, è in realtà il parlamento a mostrare oscillazioni di attenzione strettamente collegate a quelle dei media.

Questi dati oltre che confermare quanto sapevamo già sulla distribuzione degli atteggiamenti dell'opinione pubblica, segnalano come l'attenzione prestata dai partiti politici al tema bosniaco sia fortemente influenzata dagli sviluppi degli eventi in Bosnia, come riportati (e forse amplificati) dai media ${ }^{21}$. Da questo punto di vista quindi è anche plausibile ritenere che l'opinione pubblica cui i partiti politici reagiscano sia quella espressa dalle opinioni dei giornali e dei loro editorialisti.

\section{Conclusioni}

In questo saggio abbiamo argomentato come il lungo processo decisionale che ha portato infine alla partecipazione italiana alla missione Ifor in Bosnia sia avvenuto in modo sostanzialmente indipendente dagli ( $o$, forse meglio, nonostante gli) orientamenti dell'opinione pubblica italiana. Da questa prospettiva, videopolitica e democrazia governata dai sondaggi appaiono preoccupazioni ben distanti dalla realtà del policy making nazionale, per lo meno nella politica di sicurezza. Le opinioni nel pubblico e le opinioni del pubblico, per riprendere la di-

21 A rigore, un test efficace della relazione tra eventi, notizie giornalistiche ed attenzione del Parlamento richiederebbe una misura indipendente degli sviluppi in Bosnia, per accertare eventuali lag tra notizie, dibattiti parlamentari ed accadimenti politico-militari in Bosnia. In questa fase, mancando di tale misura, possiamo assumere, plausibilmente, che media e parlamento reagiscano entrambi agli eventi in Bosnia, ma i politici in maniera mediata da quanto i giornali stessi riportano. Sul ruolo dei media, in particolare la carta stampata, nella costruzione delle immagini della realtà dei politici italiani si è già detto nel secondo paragrafo. 
stinzione di Sartori (1987), appaiono così a prima vista irrilevanti agli occhi dei decisori italiani. Da una diversa prospettiva, tuttavia, la vicenda di Bosnia ha fatto a nostro avviso emergere alcuni interessanti elementi del nesso opinione pubblica-elaborazione delle politiche nel nostro paese.

Anzitutto sulla natura dell'opinione pubblica per i policymakers. Se ci poniamo la domanda: «quali sono le pubbliche opinioni che contano?» è evidente che per i politici al governo l'opinione del pubblico, una opinione cioè autonoma ed articolata, è quella dei mass media e dei parlamentari (più dei parlamentari che non dei partiti). Questi appaiono essere i veri referenti con i quali confrontarsi ed il cui consenso è opportuno garantire. Per contrasto, l'opinione nel pubblico, l'opinione cioè percepita come eteronoma, fluttuante e umorale, è invocata, quando lo è, appunto in quanto tale, e per sostenere scelte politiche coerenti con le preferenze della prima. Questo spiega il paradosso di un atteggiamento del governo italiano realista al rovescio: un governo riluttante ad intervenire non perché in contrasto con un'opinione pubblica «permissiva» bensì perché convinto della volatilità di tale orientamento permissivo.

Tuttavia la nostra analisi, per arrivare ad un secondo punto, mostra come tale volatilità dell'opinione pubblica sia più assunta che reale. Il sostegno per la partecipazione italiana si è manifestato infatti abbastanza precocemente ed è rimasto stabile nel tempo, aumentando anzi una volta che le truppe italiane sono state effettivamente schierate. Non si è trattato, in altre parole, di flussi di consenso variabili o intermittenti, non quindi di opinioni $n e l$ bensì del pubblico. Si potrebbe sostenere che siamo di fronte ad una situazione eccezionale, per la vicinanza del nostro paese all'area di crisi. Può essere possibile, ma dobbiamo anche ricordare che tale stabilità appare affatto coerente con gli andamenti riscontrati in passato che, come abbiamo segnalato nella terza sezione di questo saggio, mostrano una struttura dell'opinione pubblica italiana in politica estera tutt'altro che volatile. Inoltre i cambiamenti d'opinione, che pur avvengono, non sono così frequenti ed improvvisi, né tantomeno superiori per intensità rispetto ad altri paesi considerati stabili.

Infine, per quanto riguarda l'influenza dell'opinione pubblica sulla politica estera, ma più in generale sul policy making, la ricostruzione della vicenda bosniaca che abbiamo proposto segnala come la decisione italiana di intervenire sia sicuramente dipesa da un insieme di fattori esterni come interni, in partico- 
lare l'accordo di pace di Dayton, tra i primi, e la sopravvenuta disponibilità di un nucleo di forze armate professionali, tra i secondi. La stabile opinione nel pubblico non ha esercitato una significativa influenza poiché, male interpretata, non è stata considerata come una risorsa di legittimazione. Tuttavia riteniamo che, per l'insieme dei motivi già discussi (dal cambiamento del sistema internazionale alla minore presa delle subculture politiche sulla popolazione, dall'emergere di un bipartisan consensus sulle scelte fondamentali a un maggiore internazionalismo degli italiani) gli orientamenti dell'opinione pubblica siano destinati a esercitare una maggiore influenza sulle scelte di politica estera del nostro paese. A costituire, cioè, non solo un vincolo ma anche una risorsa per l'azione politica.

In questa direzione, la recente crisi del Kosovo ci consegna, a nostro avviso, un esempio sufficientemente chiaro di come l'opinione pubblica abbia costituito per il governo D'Alema una risorsa preziosa per attenuare i rischi di intrappolamento del governo tra calo di consenso popolare, conflittualità intra-coalizionale e imperativo della fedeltà atlantica. L'incertezza iniziale del Presidente del consiglio rifletteva infatti non solo la tradizionale difficoltà della sinistra italiana sul tema della guerra, ma anche la conoscenza del dato di fatto che non più del $40 \%$ dell'opinione pubblica - un valore comunque elevato - era favorevole ai raid della Nato. Cosciente sia dell'inevitabile calo di popolarità con il proseguire dei bombardamenti, ma anche del consenso che gli italiani esprimono nei confronti della Nato, la scelta del governo ha così coniugato il mantenimento dell'impegno militare con l'avvio di autonome iniziative diplomatiche. Su questa strada, contando soprattutto sull'effetto «annuncio» e sull'indeterminatezza riguardo l'efficacia delle autonome iniziative di pace, il governo è riuscito ad ottenere il consenso, adeguatamente pubblicizzato dai media, della maggioranza degli italiani: nel maggio 1999, una indagine Polimetrica-Swg da noi diretta riscontrava così che l'approvazione per la posizione del governo sul Kosovo oscillava tra il $57 \%$ degli elettori del Polo ed il $67 \%$ di quelli di centro-sinistra, sebbene il consenso specifico sui bombardamenti Nato non si estendesse oltre il $37 \%$ degli italiani. Piuttosto che apparire come dominato dagli eventi, la scelta del governo è stata quella di (auto)promuovere un'immagine di guida, saldamente al centro dell'azione, esprimendo leadership, effettiva o simbolica che fosse. Ciò appare in forte contrasto con lo stile di policy, reattivo e di basso profilo, emerso nel caso bosniaco. 
Questo diverso atteggiamento ha consentito, sulla base di una legittimità proveniente da un ampio consenso bipartisan dell'opinione pubblica, di imprimere (o, meglio, imporre) una maggiore coesione tra le varie componenti, alcune fortemente recalcitranti, del gabinetto, da una parte, consentendo all'Italia, dall'altra, di esprimere in sede europea e in sede Nato le proprie preferenze, e i dubbi, sulla condotta della crisi del Kosovo.

\section{Riferimenti bibliografici}

Achen, C. (1975), Mass Political Attitudes and the Survey Response, in «American Political Science Review», n. 4, pp. 1218-1231.

Almond, G.A. (1960), The American People and Foreign Policy, New York, Praeger.

Ammendola, T. (1993), Opinione pubblica e politica militare in Italia, in «Rivista Trimestrale di Scienza dell'Amministrazione», nn. 3-4, pp. 277-299.

Agnews, J. (1997), The Myth of Backward Italy in Modern Europe, in B. Allen e M. Russo (a cura di), Revisioning Italy. National Identity and Global Culture, Minneapolis, The University of Minnesota Press, pp. 23-42.

Battistelli, F. (1996), Soldati. Sociologia dei militari italiani nell'era del peace-keeping, Milano, Angeli.

Battistelli, F. e P. Isernia (1992), Dove gli angeli non osano mettere piede: opinione pubblica e politica internazionale in Italia, in «Teoria Politica», n. 1, pp. 81-119.

Bellucci, P. (1996), Opinione pubblica giovanile e difesa, in F. Battistelli (a cura di), Giovani e Forze Armate. Aspetti sociologici della condizione giovanile e della comunicazione istituzionale, Milano, Angeli.

- (1998), Difesa, politica e società. La politica militare italiana tra obiezione di coscienza e professionalizzazione delle Forze Armate, Milano, Angeli.

Bellucci, P. e P. Isernia (in stampa), Massacring in Front of a Blind Audience? Italian Public Opinion and Bosnia, in R. Sobel e E. Shiraev (a cura di), International Public Opinion and the Bosnia Crisis, Penn State University Press.

Berelson, B. (1952), Democratic Theory and Public Opinion, in «Public Opinion Quarterly», autunno, pp. 313-330.

Blumer, H. (1953), Public Opinion and Public Opinion Polling, in B. Berelson e M. Janowitz (a cura di), Reader in Public Opinion and Communication, New York, The Free Press, pp. 594-602.

Cartocci, R. (1990), Elettori in Italia. Riflessioni sulle vicende elettorali negli anni ottanta, Bologna, Il Mulino. 
Caspary, W.R. (1970), The «Mood» Theory: A Study of Public Opinion on Foreign Policy, in «American Political Science Review», n. 3, pp. 536-547.

Castronovo, V. e N. Tranfaglia (a cura di) (1995), La stampa italiana nell'età della televisione, Roma-Bari, Laterza.

Ceri, P. (a cura di) (1997), Politica e sondaggi, Torino, Rosenberg \& Sellier.

Cohen, B.C. (1977-78), Political Systems, Public Opinion and Foreign Policy: The United States and the Netherlands, in «International Journal», n. 1, pp. 195-216.

- (1995), Democracies and Foreign Policy. Public Participation in the United States and the Netherlands, Madison, University of Wisconsin Press.

Converse, J.M. (1987), Survey Research in the United States. Roots and Emergence 1890-1960, Berkeley, University of California Press.

Converse, P.E. (1964), The Nature of Belief Systems in Mass Publics, in D.E. Apter (a cura di), Ideologies and Discontent, New York, The Free Press.

- (1987), Public Opinion and the Political Process, in «Public Opinion Quarterly», inverno, S12-S24.

De Konink, M. e J. van der Meulen (1998), Zero-Dead? Testing the 'Casualty Hypothesis': Dutch Public Opinion and Peace-Keeping in Bosnia, Paper presentato al Workshop «Democracy, public opinion and the use of force in a changing international environment», Joint sessions of Workshops, Ecpr, University of Warwick, 23-28 marzo 1998.

Delli Carpini, M.X. e S. Keeter (1992), The Public's Knowledge of Politics, in J.D. Kennamer (a cura di), Public Opinion, the Press and Public Policy, Westport (Conn.), Praeger, pp. 19-40.

Eichenberg, R.C. (1998), Domestic Preferences and Foreign Policies, in «Mershon International Studies Review», suppl. 1, pp. 97-105.

Entman, R.M. (1989), Democracy without Citizens. Media and the Decay of American Politics, Oxford, Oxford University Press.

- (1998), Declarations of Independence: Media, «Pulic Opinion» and ns Foreign Policy after the Cold War, Paper presentato alla Conferenza «Public Opinion, The Mass Media and European and American Foreign Policy, The Italian Academy for Advanced Studies in America», Columbia University, 19-20 novembre.

Everts, P.E. (1996), Public Support for the Military UN-Operation in the Netherlands: The Case of Bosnia-Herzegovina. A New Look at the 'Body-Bag Hypothesis', Paper presentato alla Conferenza «Democracy, Public Opinion and the Policy of Defense», Certosa di Pontignano, Siena, 7-10 ottobre 1996.

Everts, P.E. e P. Isernia (a cura di) (in stampa), When the Going Gets Tough. Public Opinion and the Use of Force, London, Routledge. Gabel, M.J. (1998), Interests and Integration. Market Liberalization, 
Public Opinion and European Union, Ann Arbor (Mich.), University of Michigan Press.

Geer, J.G. (1996), From Tea Leaves to Opinion Polls, New York, Columbia University Press.

Graziano, L. (1968), La politica estera italiana nel dopoguerra, Milano, Marsilio.

Guidorossi, G. (1984), Gli italiani e la politica. Valori, opinioni, atteggiamenti dal dopoguerra ad oggi, Milano, Angeli.

Herbst, S. (1998), Reading Public Opinion. How Political Actors View the Democratic Process, Chicago, The University of Chicago Press.

Holmes, J. (1993), La politica estera italiana, in S. Hellman and G. Pasquino (a cura di), Politica in Italia. I fatti dell'anno e le interpretazioni, Bologna, Il Mulino, pp. 181-197.

Holsti, O.R. (1992), Public Opinion and Foreign Policy: Challenges to the Almond-Lippmann consensus, in «International Studies Quarterly», n. 4, pp. 439-466.

Hurwitz, J. e M. Peffley (1987), How Are Foreign Policy Attitudes Structured? A Hierarchical Model, in «American Political Science Review», n. 4, pp. 1099-1120.

Isernia, P. (1992), Opinione pubblica e politica di difesa in Italia, in C.M. Santoro (a cura di), L'Elmo di Scipio. Il nuovo modello di difesa, Bologna, Il Mulino, pp. 219-261.

- (1996a), Bandiera e risorse: La politica estera italiana negli anni ottanta, in M. Cotta e P. Isernia (a cura di), Il gigante dai piedi d'argilla. La crisi del regime partitocratico in Italia, Bologna, Il Mulino, pp. 139-188.

- (1996b), Dove gli angeli non mettono piede. Opinione pubblica e politiche di sicurezza in Italia, Milano, Angeli.

Isernia, P., Z. Juhasz e H. Rattinger (1998), Foreign Policy and the Rational Public in Comparative Perspective, paper presentato alla Conferenza Annuale dell'American Political Science Association, 3-6 settembre, Boston.

Jacobs, L.R. (1993), The Health of Nations. Public Opinion and the Making of American and British Health Policy, Ithaca, Cornell University Press.

Jacobs, L.R. e R.Y. Shapiro (1994), Studying Substantive Democracy, in «PS: Political Science \& Politics», n. 1, pp. 9-17.

Kinder, D.R. e D.O. Sears (1985), Public Opinion and Political Action, in A. Aronson e G. Lindzey (a cura di), Handbook of Social Psychology, New York, Random House.

Kogan, N. (1965), La politica estera italiana, Milano, Lerici.

Kull, S., I.M. Destler e C. Ramsay (1997), The Foreign Policy Gap. How Policymakers Misread the Public, A Report by the Center for International and Security Studies at the University of Maryland and the Program on International Policy Attitudes, University of Maryland. 
Lazarsfeld, P.F. (1957), Public Opinion and the Classical Tradition, in «Public Opinion Quarterly», n. 1, pp. 39-53; trad. it. in Metodologia e Ricerca Sociologica, Bologna, Il Mulino, pp. 891-914.

Leiss, A. e L. Paolozzi (1997), Come la stampa usa i sondaggi, in P. Ceri (a cura di), Politica e Sondaggi, Torino, Rosenberg \& Sellier, pp. 247-259.

Luttwak, E.N. (1994), Where Are the Great Powers? At Home with the Kids, in «Foreign Affairs», n. 4, pp. 23-28.

McCombs, M., E. Einsiedel e D. Weaver (1991), Contemporary Public Opinion. Issues and the News, Hillsdale (N.J.), Lawrence Erlbaum Ass.

Murialdi, P. (1998), La stampa italiana dalla Liberazione alla crisi di fine secolo, Roma-Bari, Laterza.

Neal, P.M. (1995), La «nuova» politica estera italiana, in P. Ignazi e R.S. Katz (a cura di), Politica in Italia. I fatti dell'anno e le interpretazioni, Bologna, Il Mulino, pp. 185-195.

Nie, N. e A. Kristi (1974), Mass Belief System Revisited: Political Change and Attitude Structure, in «Journal of Politics», agosto, pp. 540-591.

Niedermayer, O. e R. Sinnott (1995), Public Opinion and Internationalized Governance, Oxford, Oxford University Press.

Oreglia, S. (1999), L'opinione pubblica e la politica estera: un'analisi del pubblico francese in prospettiva comparata, occasional papers del Centro Interdipartimentale di Ricerca sul Cambiamento Politico (CIRCaP), n. 4, Siena.

Page, B.I. (1994), Democratic Responsiveness? Untangling the Links between Public Opinion and Policy, in «PS: Political Science \& Politics», n. 1, pp. 25-29.

Page, B.I. e R.Y. Shapiro (1982), Effects of Public Opinion on Policy, in «American Political Science Review», n. 1, pp. 175-190.

- (1992), The Rational Public. Fifty Years of Trends in American Policy Preference, Chicago, The University of Chicago Press.

Page, B.I., R.Y. Shapiro e G.R. Dempsey (1987), What Moves Public Opinion, in «American Political Science Review», n. 1, pp. 23-44.

Panebianco, A. (1989), Le strutture di rappresentanza, in L. Morlino (a cura di), Scienza Politica, Torino, Edizioni Fondazione G. Agnelli, pp. 107-143.

- (1997), Guerrieri Democratici. La democrazia e la politica di poten$z a$, Bologna, Il Mulino.

Pizzorno, A. (1980), I soggetti del pluralismo. Classi, partiti, sindacati, Bologna, Il Mulino.

Powlick, P.J. e A.Z. Katz (1998), Defining the American Public Opinion/Foreign Policy Nexus, in «Mershon International Studies Review», suppl. n. 1, pp. 29-62.

Price, V. (1992), Public Opinion, Newbury Park-London, Sage.

Putnam, R.D. (1977), Italian Foreign Policy: the Emergent Consensus, in H.R. Penniman (a cura di), Italy at the Polls. The Parliamentary 
Elections in 1976, Washington (D.C.), American Enterprise Institute, pp. 287-326.

- (1978), Interdependence and the Italian communists, in «International Organization», n. 2, pp. 301-349.

- (1988), Diplomacy and Domestic Politics: the Logic of Two-level Games, in «International Organization», n. 3, pp. 427-460.

- (1993), Making Democracy Work, Princeton (N.J.), Princeton University Press, trad. it. La tradizione civica nelle regioni italiane, Milano, Mondadori 1993.

Risse-Kappen, T. (1991), Public Opinion, Domestic Structures and Foreign Policy in Liberal Democracies, in «World Politics», n. 4, pp. 479-512.

Rosenau, J.N. (1961), Public Opinion and Foreign Policy. An Operational Formulation, New York, Random House.

Sani, G. (1974), Canali di comunicazione politica e orientamenti dell'elettorato, in «Rivista Italiana di Scienza Politica», n. 2, pp. 371-386.

- (1980), The Political Culture of Italy: Continuity and Change, in G.A. Almond e S. Verba (a cura di), The Civic Culture Revisited, London, Sage, pp. 273-325.

Sartori, G. (1987), Elementi di teoria politica, Bologna, Il Mulino.

Schelling, T.C. (19807), The Strategy of Conflict, Cambridge (Mass.), Harvard University Press.

Sciolla, L. (1990), Identità e mutamento culturale nell'Italia di oggi, in V. Cesareo (a cura di), La cultura dell'Italia contemporanea. Trasformazione dei modelli di comportamento e identità sociale, Torino, Fondazione G. Agnelli.

Segatti, P. (1997), Gli orientamenti dei giovani in Italia e in Europa, in R. Cartocci e A.M.L. Parisi (a cura di), Difesa della patria e interesse nazionale nella scuola, Milano, Angeli, pp. 87-146.

Shapiro, R.Y. e L.R. Jacobs (1989), The Relationship between Public Opinion and Public Policy: a Review, in S. Long (a cura di), Political Behavior Annual, vol. 2, Boulder (Colo.), Westview Press, pp. 149-179.

Shapiro, R.Y. e B.I. Page (1988), Foreign Policy and the Rational Public, in «Journal of Conflict Resolution», n. 2, pp. 211-247.

Sobel, R. (1996), US and European Attitudes toward Intervention in the Former Yugoslavia: Mourir pour la Bosnie, in R.H. Ullman (a cura di), The World and Yugoslavia's Wars, New York, Council of Foreign Relations Books, pp. 145-181.

Tullio-Altan, C. (1986), La nostra Italia. Arretratezza culturale, clientelismo, trasformismo e ribellione dall'Unità ad oggi, Milano, Feltrinelli. 